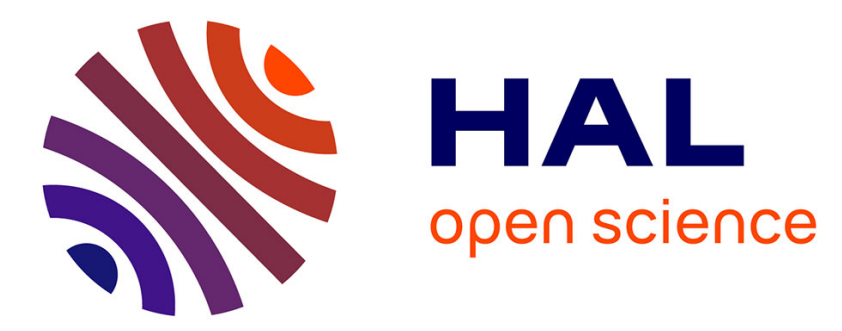

\title{
Martian Infrasound: Numerical Modeling and Analysis of InSight's Data
}

Léo Martire, R. F Garcia, Lucie Rolland, A Spiga, Philippe Lognonné, Don Banfield, William Bruce B Banerdt, Roland Martin

\section{- To cite this version:}

Léo Martire, R. F Garcia, Lucie Rolland, A Spiga, Philippe Lognonné, et al.. Martian Infrasound: Numerical Modeling and Analysis of InSight's Data. Journal of Geophysical Research. Planets, 2020, 125 (6), 10.1029/2020JE006376 . hal-02971385

\section{HAL Id: hal-02971385 \\ https://hal.science/hal-02971385}

Submitted on 19 Oct 2020

HAL is a multi-disciplinary open access archive for the deposit and dissemination of scientific research documents, whether they are published or not. The documents may come from teaching and research institutions in France or abroad, or from public or private research centers.
L'archive ouverte pluridisciplinaire HAL, est destinée au dépôt et à la diffusion de documents scientifiques de niveau recherche, publiés ou non, émanant des établissements d'enseignement et de recherche français ou étrangers, des laboratoires publics ou privés. 


\title{
Martian Infrasound: Numerical Modeling and Analysis of InSight's Data
}

\author{
Léo Martire ${ }^{1}$, R. F. Garcia ${ }^{1}$, L. Rolland ${ }^{2}$, A. Spiga ${ }^{3,4}$, \\ P. Lognonné ${ }^{5}$, D. Banfield ${ }^{6}$, W. B. Banerdt ${ }^{7}$, R. Martin $^{8}$ \\ ${ }^{1}$ SSPA, Institut Supérieur de l'Aéronautique et de l'Espace - Supaero, 10 Avenue Edouard Belin, Toulouse, \\ Occitanie, 31400, France. ${ }^{2}$ Université Côte d'Azur, Observatoire de la Côte d'Azur, Centre National de la \\ Recherche Scientifique (CNRS), Nice, Provence-Alpes-Côte d'Azur, 06300, France. ${ }^{3}$ Laboratoire de Météorologie \\ Dynamique (LMD) / Institut Pierre-Simon Laplace (IPSL), Sorbonne Université, Centre National de la \\ Recherche Scientifique (CNRS), École Polytechnique, École Normale Supérieure (ENS), Paris, Île-de-France, \\ France. ${ }^{4}$ Institut Universitaire de France, Paris, Île-de-France, France. ${ }^{5}$ Université de Paris, Institut de Physique \\ du Globe de Paris, Centre National de la Recherche Scientifique (CNRS), Paris, Île-de-France, France. ${ }^{6}$ Cornell \\ Center for Astrophysics and Planetary Science (CCAPS), Cornell University, Ithaca, New York, USA. ${ }^{7}$ Jet \\ Propulsion Laboratory (JPL) / California Institute of Technology (Caltech), Pasadena, California, USA. \\ ${ }^{8}$ Géosciences Environnement Toulouse (GET), Observatoire Midi-Pyrénées (OMP), 14 Avenue Edouard Belin, \\ Toulouse, Occitanie, 31400, France.
}

Accepted for publication in Journal of Geophysical Research: Planets. An edited version of this paper was published by AGU.

Copyright (2020) American Geophysical Union.

Further reproduction or electronic distribution is not permitted.

Léo Martire, R. F. Garcia, L. Rolland, A. Spiga,

P. Lognonné, D. Banfield, W. B. Banerdt, R. Martin (2020).

Martian Infrasound: Numerical Modeling and Analysis of InSight's Data. Journal of Geophysical Research: Planets. dx.doi.org/10.1029/2020JE006376

\section{Key Points:}

- Our numerical modeling predicts both pressure variations and ground movements induced by Martian acoustic waves.

- Regional propagation of dispersed infrasound is expected in the Martian nocturnal surface waveguide.

- Some monotone events detected by SEIS are consistent with acoustic waves trapped in this waveguide.

Corresponding author: Léo Martire, leo.martire@isae.fr 


\begin{abstract}
Acoustic waves in planetary atmospheres couple into the solid surface, producing ground displacements that can be measured using seismometers. On November 26 2018, the InSight mission successfully landed on Mars. Its objectives include studying Mars' interior using the seismometer SEIS (Seismic Experiment for Interior Structures) and the atmosphere through the weather station APSS (Auxiliary Payload Sensor Suite). Because InSight is the first mission capable of studying infrasound on Mars, we investigate the signature of infrasound both in terms of air pressure and ground velocities. Using numerical simulations, we characterize (1) the acoustic propagation pattern in Martian dusk, and (2) the mechanical atmosphere-to-ground coupling under acoustic waves. Then, using SEIS data, we demonstrate that two low-frequency monotone events (S0133a and S0189a) are in fact infrasound trapped in the atmospheric nocturnal surface waveguide. We base our demonstration on the following facts. (1) Seismic signals rarely produce, at a given station, a single frequency varying from one event to the other. (2) No clear seismic phases have been identified for such events. (3) The observed SEIS signals present the characteristics expected for trapped infrasound observed through their compliance effects (specific frequency response, more energy on the vertical component, $\pm 90^{\circ}$ phase shift between vertical and horizontal components, no detection on pressure sensor at these low amplitude levels). Our simulations of the nocturnal waveguide's response is however subject to uncertainties because 1) it relies on the sol-to-sol variability of the atmosphere, and 2) sub-surface properties are not properly known at this time.
\end{abstract}

\title{
Plain Language Summary
}

The InSight mission landed on Mars the 26/11/2018 and was designed to study the interior of the Red Planet. The noise level of its seismometer SEIS routinely stays under $1 \mathrm{~nm} / \mathrm{s}^{2}$, a precision unmatched by other planetary seismometers. InSight also features a meteorological station, APSS, able to measure the atmosphere's absolute pressure, wind, and temperature. In this work, we use the seismometer and meteorological station to investigate acoustic waves (sound) in the Martian atmosphere.

Those acoustic waves are pressure perturbations, and consequently cause the ground to move: positive perturbations push the soil down, whereas negative ones lift it up. InSight's SEIS and APSS constitute the perfect instruments for investigating ground-coupled acoustic waves.

We start by performing numerical simulations of the coupled solid-atmosphere system. In particular, we investigate how low-frequency sound (infrasound) travels in Mars' atmosphere. We then derive key properties of the ground deformations due to passing infrasound. These arguments are then applied to InSight's data: ground motion measurements from SEIS and pressure records from APSS.

Finally, we show that 3-axis ground motion can help discriminate acoustic waves from meteorological perturbations. We also demonstrate that some SEIS events are caused by infrasound trapped close to the surface by nocturnal winds.

\section{Introduction}

The InSight mission landed in Elysium Planitia on Mars on the 26th of November, 2018. The full multi-purpose instrument suite is designed to explore the interior of the red planet. Notably, the seismometer SEIS (Seismic Experiment for Interior Structure) is able to measure ground movements with exceptional precision (Lognonné et al., 2019, 2020). Moreover, the weather station at the surface of Mars onboard InSight APSS, the Auxiliary Payload Sensor Suite - records the ambient pressure as well as the wind strength and direction, with unprecedented continuity, sampling frequency (for pressure) and sensitivity (Spiga et al., 2018; Banfield et al., 2019, 2020). 
Acoustic signals are thought to propagate in the atmosphere of Mars according to theoretical studies (Bass \& Chambers, 2001; Williams, 2001). Both its seismometer and pressure sensor make InSight capable, for the first time, to detect on Mars signals belonging to the lower-frequency acoustic range, i.e., infrasonic signals (Gossard \& Hooke, 1975; Evers \& Haak, 2009). If it was not for the low-pass filtering due to atmospheric carbon dioxide $\left(\mathrm{CO}_{2}\right)$, SEIS could in principle record ground-coupled infrasonic waves from $0.01 \mathrm{~Hz}$ up to its sampling limit of $50 \mathrm{~Hz}$. Candidate infrasound signals have been detected by InSight's pressure sensor (Banfield et al., 2020) and are in need to be confirmed by further analysis.

The ground-atmosphere system is mechanically coupled. This enables atmospheric acoustic waves to be converted into ground motion (and vice versa). Two main types of air-to-ground conversion coexist: classical acoustic wave to seismic wave transmission (Aki \& Richards, 2002) and compliance effects (Sorrells et al., 1971; Sorrells, 1971; Kenda et al., 2017). A smooth transition links the two (Woods et al., 2015). Additionally, studying the ground displacements caused by infrasonic waves enables one to constrain the sub-surface below the InSight lander.

It can be challenging to distinguish between acoustic waves and other atmospheric phenomena using a single pressure sensor, since the propagation speeds of the disturbances cannot be determined. Analyzing the ground motion induced by these perturbations allows one to differentiate various phenomena. The main contribution of this paper is demonstrating that we can perform this discrimination using the seismometer SEIS onboard InSight.

Understanding acoustic waves' propagation patterns also helps to sound the atmospheric wind structure and can yield valuable information on distant atmospheric events (Drob et al., 2003). Particularly on Mars, it may allow the remote investigation of meteor entries (Williams, 2001) and related impacts (Daubar et al., 2018), as well as possible nearby landslides, rockfalls, or ejecta falls. Based on terrestrial analogues, one can also reasonably expect acoustic signals from spacecraft entry (Garcés et al., 2004; Yamamoto et al., 2011), the seismo-acoustic coupling of marsquakes (Krishnamoorthy et al., 2018; Martire et al., 2018; Krishnamoorthy et al., 2019), dust devils (Tatom et al., 1995; Bedard, 2005; Lorenz \& Christie, 2015), wind-mountain interactions (Larson et al., 1971), and atmospheric turbulence (Howe, 2002).

Furthermore, again according to Earth's experience, the major sources of atmospheric gravity waves, namely topography, the geostrophic adjustment in jets and fronts, and convective storms, may act as sources of infrasound (Campus \& Christie, 2009). All those infrasound sources are expected to be active in the Martian environment, including the convective-storm source in dust storms (Heavens et al., 2019) and the nighttime water-ice clouds (Spiga et al., 2017). The abrupt transition between daytime and nighttime on Mars (Savijärvi \& Siili, 1993; Haberle et al., 1993), conducive to significant changes at both regional scales (slope winds) and local scales (collapse of daytime buoyancy-driven convection in the planetary boundary layer and arising of the nocturnal low-level jets) could also be a possible source of infrasound on Mars.

Finally, given the overarching goal of the InSight mission to probe the interior of Mars, understanding the atmospheric dynamics causing infrasound is key to decoupling pressure-induced effects from seismic signals.

Atmospheric ducts are atmospheric layers where acoustic waves can be refracted back due to changes in the effective sound speed gradient (Whitaker \& Norris, 2008). This behavior can be due either to the thermal structure of the atmosphere or to the presence of horizontal winds. If close to the ground, the refracted acoustic waves also reflect on the surface, and subsequently become trapped in the low atmosphere acting as a waveguide. Hence, such waveguide constitutes a favorable layer for ground-toground infrasound propagation over large distances (Drob et al., 2003; Garcia et al., 
2017). Additionally, if the duct is particularly narrow, constructive interference can produce dispersive modes (Herrin et al., 2006; Negraru \& Herrin, 2009).

We particularly focus on the waveguides in the lower $(\leq 20 \mathrm{~km}$ altitude) Martian atmosphere (Spiga et al., 2018). To be specific, we will distinguish two chief types of structures.

1. The large-scale waveguide is due to the high-altitude wind jets, themselves linked to the global atmospheric circulation. On Earth, these winds correspond to the stratospheric jets existing around $50 \mathrm{~km}$ altitude (Le Pichon et al., 2005). Mars being devoid of a stratosphere, it exhibits instead a tropospheric waveguide which is located at altitudes $>10-15 \mathrm{~km}$ above the surface, where wind speeds increase significantly on the global scale.

2. The waveguide close to the surface is due to the regional, low-level wind jet appearing at night within the planetary boundary layer. This phenomenon is common on Earth, though confined to a few hundred meters above the surface only and thus much more subject to surface roughness (Garratt, 1992; Waxler et al., 2008). There have been a few terrestrial observations of infrasound trapped very close to the surface, showcasing dispersive wavetrains (Herrin et al., 2006; Negraru \& Herrin, 2009). Mars being also prone to strong nocturnal low-level jets at altitudes 1-2 km above the surface (Savijärvi \& Siili, 1993), we call this duct the nocturnal waveguide.

Note that acoustic ducts also exist at higher altitudes. We choose not to investigate them here, since they are expected to undergo much more attenuation (due to the $\mathrm{CO}_{2}$-rich atmosphere).

This paper first presents in Section 2 an overview of atmospheric pressure perturbations. Their effect on the ground motion is detailed, with an emphasis on the main air-to-ground conversion modes. Then, Section 3 presents the numerical simulation tools, which are applied to the Martian case in Section 4. Synthetic results are presented, with a focus on infrasound trapped in the nocturnal waveguide. Next, in Section 5, a selection of events recorded by SEIS are considered. We demonstrate that two of them are not seismic but rather caused by trapped infrasound. Finally, conclusions are drawn in Section 6, alongside possible future work.

\section{Atmospheric Pressure Perturbations and Conversion to Ground Mo- tion}

\subsection{Atmospheric Pressure Perturbations on Mars}

Atmospheric dynamics induce various types of atmospheric pressure perturbations at different wavelength scales and frequencies (Banfield et al., 2020; Spiga et al., 2018). It is relevant to consider the pressure perturbations in SEIS' main frequency range, that is between $1 \mathrm{mHz}$ and $50 \mathrm{~Hz}$ (Lognonné et al., 2019, 2020). This range covers gravity waves, infrasonic waves, and the various pressure perturbations induced by atmospheric dynamics. In this contribution, we focus only on the two latter, which exhibit very distinctive characteristics.

On one hand, the atmosphere's dynamics are governed by fluid mechanics or turbulence equations, and consequently the subsequent pressure perturbations generally move horizontally at wind speed $c_{\mathrm{w}}$. On the other hand, infrasonic signals are governed by the acoustic wave equations and dispersion relation, and propagate at first order at the effective speed of sound $c_{\mathrm{eff}}=c_{\mathrm{s}}+c_{\mathrm{w}}$, with $c_{\mathrm{s}}$ the speed of sound.

Noting the effective sound speed is an approximation, we only give it for comparing orders of magnitude of propagation speeds. It is obtained by neglecting terms in $\left(\mathrm{Ma}^{p}\right)_{p \geq 2}$ (Ma the Mach number) in the wave equation, as pointed out for instance 
by Assink (2012) in his Appendix A.4 or by Godin et al. (1993). Remaining general, one can define the full vector sound velocity in a given direction $\hat{n}, \vec{c}(\hat{n})$, as:

$$
\vec{c}(\hat{n})=\sqrt{\gamma R_{\mathrm{sp}} T} \hat{n}+\vec{u},
$$

with $\hat{n}$ the unit vector in the desired direction, $\gamma$ the adiabatic index, $R_{\mathrm{sp}}$ the specific gas constant, $T$ the temperature, and $\vec{u}$ the wind vector. Remark that $\sqrt{\gamma R_{\mathrm{sp}} T}$ is the isotropic thermodynamic speed of sound $c_{\mathrm{s}}$. Along a given direction or azimuth, $\vec{u}$ reduces to $c_{\mathrm{w}}$, and $\vec{c}(\hat{n})$ to $c_{\mathrm{eff}}$.

In this paper, we narrow our focus to infrasonic waves in the $0.05-2 \mathrm{~Hz}$ frequency range. This range is limited for the lower frequencies by the atmospheric acoustic cutoff frequency $\left(f_{\mathrm{c}, \mathrm{a}} \simeq c_{\mathrm{s}} /(2 H)\right.$ with $H$ the scale height, hence $f_{\mathrm{c}, \mathrm{a}} \simeq 240 /(2 \times 2400)=$ $0.05 \mathrm{~Hz})$. The upper bound is set according to the attenuation effects mainly induced by $\mathrm{CO}_{2}$ molecules (Bass \& Chambers, 2001; Williams, 2001; Petculescu \& Lueptow, 2007). In the 0.01-100 Hz frequency range, all intrinsic attenuation factors (aside from geometric spreading and reflection losses) are proportional to $f^{2}$. Based on the atmospheric conditions typically found at the InSight landing site between the surface and $20 \mathrm{~km}$ altitude (e.g., using the Mars Climate Database, MCD, by Forget et al. (1999) and Millour et al. (2018)), at $1 \mathrm{~Hz}$, the classical and rotational losses are at most $0.01 \mathrm{~dB} / 100 \mathrm{~km}$. In contrast, the vibrational attenuation losses due to $\mathrm{CO}_{2}$ are above $2 \mathrm{~dB} / 100 \mathrm{~km}$.

Atmospheric pressure perturbations propagating in the coupled atmosphere/solid system cause various types of ground motion. Two main coupling processes are competing: either wave-wave transmission from fluid to solid (Aki \& Richards, 2002), or compliance effects (Sorrells et al., 1971; Sorrells, 1971). The next Sections detail the different acoustic coupling mechanisms.

\subsection{General Air-to-Ground Energy Transmission and the Wave-Wave Mode}

We start by recalling that $\mathrm{P}$-waves are compressional waves; and that $\mathrm{S}$-waves are shear elastic waves (nonexistent in fluids). These two notions are needed for the following developments.

Consider an incident acoustic plane pressure wave (a P-wave in the fluid domain), encountering some solid material at some incidence angle $0^{\circ} \leq i \leq 90^{\circ}\left(0^{\circ}\right.$ being fully orthogonal, and $90^{\circ}$ being fully parallel). It is well-known that, in the infinite-frequency ray theory limit, no energy can be transmitted across the fluid/solid interface above some critical angles. Namely, the ray conversions follow Snell's law at the interface:

$$
\frac{\sin (i)}{c_{\mathrm{s}}}=\frac{\sin \left(i_{\mathrm{p}}\right)}{v_{\mathrm{p}}}=\frac{\sin \left(i_{\mathrm{s}}\right)}{v_{\mathrm{s}}},
$$

where $c_{\mathrm{S}}$ is the atmospheric speed of sound ( $\mathrm{P}$-wave velocity in the fluid domain), and $v_{\mathrm{p}}$ (resp. $v_{\mathrm{s}}$ ) is the $\mathrm{P}$-wave velocity (resp. S-wave velocity) in the solid domain. We will assume in the remaining of this Section that $c_{\mathrm{s}}<v_{\mathrm{s}}<v_{\mathrm{p}}$, which holds for our applications. The angles $i, i_{\mathrm{p}}$, and $i_{\mathrm{s}}$ are defined from the normal vector to the interface.

According to Eq. (2), transmitted P-waves cannot exist above the critical angle for longitudinal waves, $i^{\mathrm{c}, \mathrm{p}}$, defined as:

$$
i^{\mathrm{c}, \mathrm{p}}=\arcsin \left(c_{\mathrm{s}} / v_{\mathrm{p}}\right),
$$

and transmitted S-waves cannot exist above the shear wave critical angle, $i^{\mathrm{c}, \mathrm{s}}$ :

$$
i^{\mathrm{c}, \mathrm{s}}=\arcsin \left(c_{\mathrm{s}} / v_{\mathrm{s}}\right) \text {. }
$$


Since $c_{\mathrm{s}}<v_{\mathrm{s}}<v_{\mathrm{p}}$, the aforementioned angles are always ordered as $0 \leq i^{\mathrm{c}, \mathrm{p}}<i^{\mathrm{c}, \mathrm{s}}<$ $90^{\circ}$. Consequently, acoustic waves may only convert to seismic waves for incidences $i$ lower than a wave-wave critical angle, $i^{\mathrm{c}}$, which we define as:

$$
i^{\mathrm{c}}=\max \left(i^{\mathrm{c}, \mathrm{p}}, i^{\mathrm{c}, \mathrm{s}}\right)=i^{\mathrm{c}, \mathrm{s}}=\arcsin \left(c_{\mathrm{s}} / v_{\mathrm{s}}\right) .
$$

We call energy transmissions occurring at incidences $i<i^{\mathrm{c}}$ the wave-wave mode. Additionally, note that the pressure perturbations $\Delta P$ are necessarily in phase with the subsequent ground vertical velocity $V_{z}$.

However, general decaying and propagating incident acoustic waves above the wave-wave critical angle $\left(i>i^{\mathrm{c}}\right)$ can still produce an evanescent ground motion disturbance (Woods et al., 2015). In their Eq. (21), Woods et al. (2015) show the ground motion can still be nonzero for high incidence angles. Their Figure 9 showcases the transmitted intensity and the decaying of subsequent ground motion. We shall refer to this transition as the evanescent regime.

Finally, one can consider incidence angles rising to $i=90^{\circ}$, which is the case of fully horizontally-propagating acoustic waves. This is what we will consider in Section 2.3, and call the compliance mode.

To summarize, acoustic energy may be transmitted to the ground following various, continuously linked modes. If the incident angle $i$ verifies $0 \leq i<i^{\mathrm{c}, \mathrm{p}}$, acoustic waves can produce both $\mathrm{P}$ - and $\mathrm{S}$-waves. If $i^{\mathrm{c}, \mathrm{p}}<i<i^{\mathrm{c}, \mathrm{s}}$, only transmitted S-waves can exist. In the $i^{\mathrm{c}, \mathrm{s}}<i<90^{\circ}$ range, only an evanescent ground motion is produced (Woods et al., 2015). Finally, the limit case of $i \rightarrow 90^{\circ}$ is the compliance mode, detailed in Section 2.3.

\subsection{Theory of Ground Motion Induced by Compliance Effects}

Pressure perturbations, with a coherent pattern over horizontal scales larger than the apparent horizontal wavelength, induce ground motion through the elastic response of the ground. These compliance effects cover both the pressure perturbations stemming from atmospheric dynamics, and infrasound propagating horizontally. Such effects have been first described under a plane wave approximation by Sorrells et al. (1971). They were more recently applied within the framework of the InSight mission for ground movements induced by dust devils (Murdoch et al., 2017; Kenda et al., $2017,2020)$.

In the next Sections, we detail these compliance effects. Section 2.3.1 presents precisely how they affect SEIS' measurements. In Section 2.3.2, we give the compliance relations in the case of a homogeneous sub-surface; while we detail in Section 2.3.3 the case of stratified sub-surfaces.

\subsubsection{Types of Signals Induced on a Seismometer by Compliance Ef- fects}

Compliance effects cause three types of signals on a seismometer's sensors:

- Inertial effects generate pure ground translation along the horizontal and vertical directions.

- Tilt effects generate rotations of the ground, and consequently of the whole seismometer. Hence they are projecting the local gravity vector on the horizontal components. Since these effects are proportional to an acceleration, the $\mathrm{V} / \mathrm{P}$ ratio decreases with increasing frequency.

- Rotation effects generate rotations of both the ground and the seismometer, around the center of gravity of the latter. These effects depend on the position 
of each sensor relative to said center of gravity. They are proportional to displacement, hence the $\mathrm{V} / \mathrm{P}$ ratio increases with increasing frequency. However, they are typically only significant above $\simeq 2 \mathrm{~Hz}$.

We recall that all compliance effects are instantaneous elastic ground deformations, which are generating no seismic wave. Both infrasonic waves propagating far over the wave-wave critical angle $i^{\text {c }}$ (with incidences $i \rightarrow 90^{\circ}$, see Section 2.2) and meteorological wind-advected pressure perturbations generate ground deformations through compliance effects.

It is to be noted that buried seismometers are mechanically much less sensitive to spurious atmospheric effects (e.g., wind). However, 1) such setup remains impracticable on robotic planetary missions, and 2) the near subsurface nonetheless responds to energy transfers from the atmospheric pressure perturbations.

\subsubsection{Analytical Compliance Relations for an Homogeneous Sub-Surface Model}

The various compliance effects described in Section 2.3.1 can be estimated by analytical relations between ground velocities and pressure perturbations for an homogeneous sub-surface model and assuming a plane wave pressure perturbation moving at some horizontal speed $c$ (Sorrells et al., 1971).

Here, we set the horizontal component of ground velocity $\left(V_{h}\right)$ as positive in the direction of propagation of the moving pressure perturbation, such that the propagation speed $c>0$. Its vertical component $\left(V_{z}\right)$ is set positive when upwards. Superscripts denote the different contributions to each velocity component: $V_{h, z}{ }^{\text {i }}$ denotes the inertial effects, and $V_{h}^{\mathrm{t}}$ the tilt effects. Rotation effects are neglected here because they are only significant above $\simeq 2 \mathrm{~Hz}$.

Inertial effects on the vertical $\left(V_{z}{ }^{\mathrm{i}}\right)$ and horizontal $\left(V_{h}{ }^{\mathrm{i}}\right)$ ground velocities are linked to the pressure perturbations $(\Delta P)$ by (Kenda et al., 2017, 2020):

$$
\begin{gathered}
V_{z}^{\mathrm{i}}(f)=-2 i c \frac{1-\nu^{2}}{\mathrm{E}} \Delta P(f) \\
V_{h}^{\mathrm{i}}(f)=-c \frac{(1+\nu)(1-2 \nu)}{\mathrm{E}} \Delta P(f)
\end{gathered}
$$

where $f$ is frequency, $c$ is the horizontal propagation speed of the atmospheric pressure perturbation $\Delta P, i$ is the pure imaginary complex number, and $\nu$ and $\mathrm{E}$ are respectively the Poisson ratio and the Young's modulus of the ground. We give the full derivation of those expressions in Appendix B.

Instrument tilt effects on the horizontal component are defined by (Kenda et al., 2017, 2020):

$$
V_{h}^{\mathrm{t}}(f)=\frac{g}{\pi f} \frac{1-\nu^{2}}{\mathrm{E}} \Delta P(f)
$$

where, in addition to the previously defined variables, $g$ is the Martian gravity. The full derivation of this expression is also given in Appendix B.

Remark that the vertical (resp. horizontal) ground velocity has a $-90^{\circ}$ phase shift (resp. is in phase) relative to pressure perturbations (see (6) and (7)). In other words:

$$
\begin{gathered}
\arg \left(V_{z}{ }^{\mathrm{i}}\right)=\arg (\Delta P)-90^{\circ}, \\
\arg \left(V_{h}^{\mathrm{i}}\right)=\arg (\Delta P) .
\end{gathered}
$$

Inertial effects are proportional to the propagation speed, typically lying between wind speed $c_{\mathrm{w}} \leq 30 \mathrm{~m} / \mathrm{s}$ and acoustic speeds $200 \mathrm{~m} / \mathrm{s} \leq c_{\mathrm{eff}} \leq 270 \mathrm{~m} / \mathrm{s}$. They are 
inversely proportional to the material's Young's modulus $E$, which is estimated around InSight to rise as depth increases from $E \simeq 0.1 \mathrm{GPa}(e . g$. , sand) to $E \simeq 100 \mathrm{GPa}$ (e.g., granite).

\subsubsection{Compliance Effects for Stratified Sub-Surfaces}

In reality, the ground is not homogeneous: when considering layered soil, the compliance value depends on frequency. Since lower frequencies translate into larger wavelengths, the longer the period of the acoustic wave, the deeper it sounds the subsurface. Hence the harder the materials encountered, and consequently the lower the compliance becomes (see Eqs. (6) and (7)).

Figure 1 illustrates the inertial effects by presenting compliance estimates for three sub-surface models and various atmospheric horizontal speeds. On one hand, they can be computed for homogeneous sub-surface models using Eqs. (6) and (7). On the other hand, a more advanced method (developed by Kenda et al. $(2017,2020)$ ) can compute the compliance for layered models.

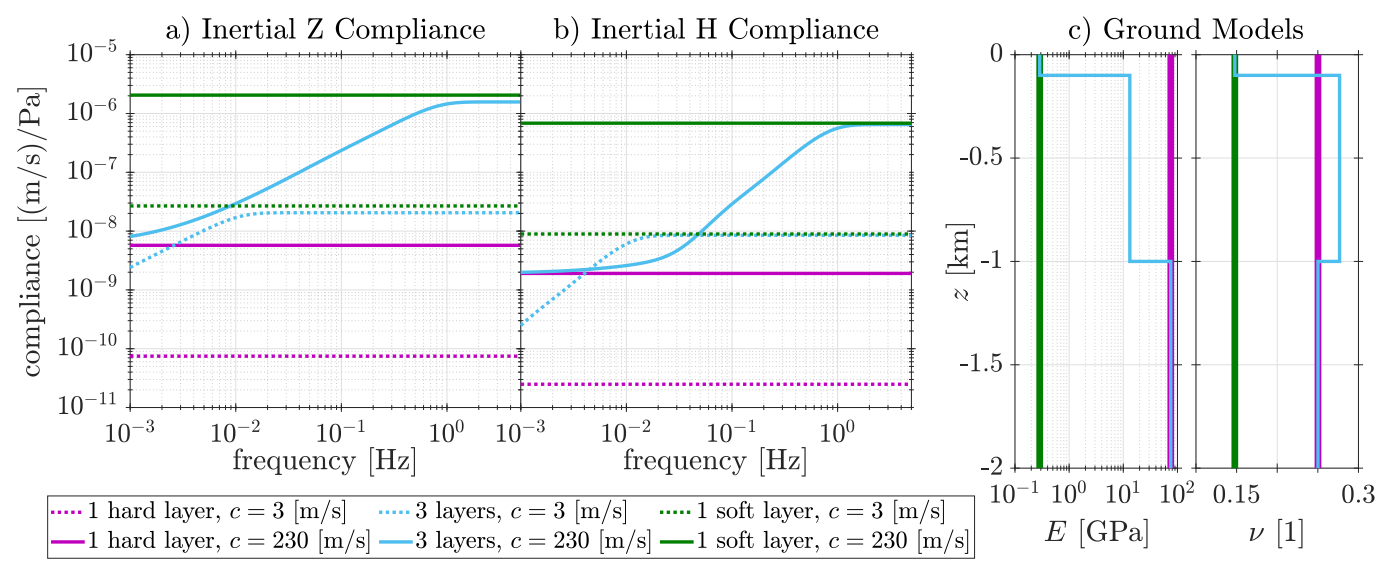

Figure 1. Compliance inertial effects' gain, as a function of frequency, for either two homogeneous 1-layer sub-surface models, or a 3-layers one. a) \& b) Inertial effects stemming from pressure perturbations are presented, along the vertical $\left.\left(\left[V_{z}^{\mathrm{i}} / \Delta P\right](f), \mathrm{a}\right)\right)$ and horizontal $\left.\left(\left[V_{x}^{\mathrm{i}} / \Delta P\right](f), \mathrm{b}\right)\right)$ axes. Solid lines: compliance for acoustic waves at effective sound speed $(230 \mathrm{~m} / \mathrm{s})$. Dashed lines: for atmospheric pressure perturbations at wind speed $(3 \mathrm{~m} / \mathrm{s}) . \mathrm{c})$ Ground models in terms of Young's modulus $E$ and Poisson coefficient $\nu$. Purple lines: hard homogeneous model. Blue lines: layered model. Green lines: soft homogeneous model. Values for the homogeneous models can be computed using Eqs. (6) and (7). For stratified models, we refer to Kenda et al. (2017, 2020).

\subsection{Compliance Effects in a Theoretical Martian Case}

In this Section, we insert realistic Martian values into the relations introduced before, in order to obtain some quantitative characteristics of compliance conversion effects. Let us consider a pre-landing sub-surface model at the InSight landing site (Delage et al., 2017; Morgan et al., 2018). It is plotted in terms of Young's modulus and Poisson ratio in Figure 1 (as the "3-layers model"), or in terms of seismic velocities in Appendix Figure A3. We will also assume that the speed of sound $c_{\mathrm{s}} \simeq 230 \mathrm{~m} / \mathrm{s}$, and that wind speed $c_{\mathrm{w}} \ll c_{\mathrm{S}}$. In these conditions, note that the critical angle $i^{\mathrm{c}} \simeq 60^{\circ}$. 
Inserting these values into the relations from Eqs. (6)-(7)-(8), we deduce the following characteristics in the $0.01-2 \mathrm{~Hz}$ frequency range.

Compliance effects for acoustic waves are dominated by inertial effects on both vertical and horizontal components. The ratio between vertical and horizontal ground velocities depends only on the Poisson ratio of the sub-surface (see Eqs. (6)-(7)), and typically equals 2.6-3 for Poisson ratios between 0.2-0.25 (Delage et al., 2017; Morgan et al., 2018). The absolute amplitude of compliance depends on the Young's modulus sensed by the acoustic wave, which strongly depends on its horizontal wavelength (see Section 2.3.3).

The inertial compliance effects associated to meteorological pressure perturbations are much lower, due to them moving at wind speed $c_{\mathrm{w}}$ instead of at effective sound speed $c_{\text {eff }}$. This arises directly from the $c$ factor in Eqs. (6)-(7). However, due to the wavelengths being shorter, this type of pressure forcing is only sensitive to the shallow sub-surface with soft properties, and thus cause large ground deformations. For this type of pressure forcing, tilt effects are dominating for the horizontal components of SEIS at frequencies below about $0.2 \mathrm{~Hz}$, whereas inertial effects are dominant on the vertical components. The ratio between vertical and horizontal components is strongly varying with frequency due to the increase of horizontal compliance induced by tilt effects with the inverse of the frequency (Eq. (8)).

\subsection{Acoustic Inertial Compliance Estimation from SEIS/APSS Data}

In this Section we focus on determining, from InSight's SEIS/APSS data, the compliance inertial values for acoustic waves. Our current knowledge of the subsurface mechanical properties below InSight's instruments is still subject to significant uncertainties. Consequently, it does not allow us to compute with reasonable accuracy the compliance expected for acoustic waves. At a given frequency, infrasound has a much larger horizontal wavelength than the small-scale meteorological pressure perturbations (e.g., dust devils or turbulence), and thus are sensitive to the mechanical properties of the sub-surface at larger depths. The typical propagation speeds may range from wind speed $\left(c_{\mathrm{w}} \leq 30 \mathrm{~m} / \mathrm{s}\right)$ to effective acoustic speeds $\left(200 \mathrm{~m} / \mathrm{s} \leq c_{\mathrm{eff}} \leq\right.$ $270 \mathrm{~m} / \mathrm{s}$ ); and the typical wavelengths from $10 \mathrm{~m}$ (small-scale meteorological perturbations) to $3 \mathrm{~km}$ (low-frequency infrasound).

That being said, the vertical compliance normalized to the horizontal speed of the wave depends only on the horizontal wavelength of the wave (Kenda et al., 2017, 2020). Consequently, we can extrapolate from atmospheric vertical compliance measurements some values for the acoustic vertical compliance, keeping the horizontal wavelength constant. To do so, we use the following formula assuming that vertical compliance normalized to horizontal speed of the wave is identical for acoustic waves and atmospheric pressure perturbations of the same wavelength:

$$
\left[\frac{V_{z}^{\mathrm{s}}}{P^{\mathrm{s}}}\right](f)=\frac{c_{\mathrm{eff}}}{c_{\mathrm{w}}} \times\left[\frac{V_{z}^{\mathrm{w}}}{P^{\mathrm{w}}}\right]\left(\frac{c_{\mathrm{w}}}{c_{\mathrm{eff}}} f\right),
$$

with $f$ the acoustic wave frequency, $c_{\text {eff }}$ the effective sound speed, $c_{\mathrm{w}}$ the wind speed, $\left[V_{z}^{\mathrm{s}} / P^{\mathrm{s}}\right](f)$ the frequency-dependent acoustic conversion ratio, and $\left[V_{z}^{\mathrm{w}} / P^{\mathrm{w}}\right](f)$ the frequency-dependent atmospheric dynamics' conversion ratio obtained by Garcia et al. (2020). Note that the $\mathrm{V} / \mathrm{P}$ ratios we will call compliance conversion coefficient in the remaining of this paper (which unit is the $(\mathrm{m} / \mathrm{s}) / \mathrm{Pa}$ ) can be understood as the inverse of the specific acoustic impedance (given in $\mathrm{Pa} \cdot \mathrm{s} / \mathrm{m}$ ).

Figure 2 presents the normalized vertical compliance obtained from atmospheric pressure perturbations (Garcia et al., 2020) and its translation into vertical compliance for acoustic waves. Because for the same horizontal wavelength, atmospheric pressure perturbations have a much lower frequency than acoustic waves, the acoustic compli- 
ance estimates are provided only above $2 \mathrm{~Hz}$. In order to have rough estimates of their values in the $0.4-1 \mathrm{~Hz}$ range, we fit a power law model, implicitly assuming that the sub-surface's Young's modulus continues to increase with depth.

Despite this strong assumption, this model is critical to deciphering between the acoustic waves and the atmospheric pressure perturbations from the vertical ground velocities measured by SEIS. This compliance power law is a lower bound of the expected acoustic compliance, because we expect the compliance to reach a plateau at low frequencies, i.e., when the wavelength is long enough to overlap with bedrock.
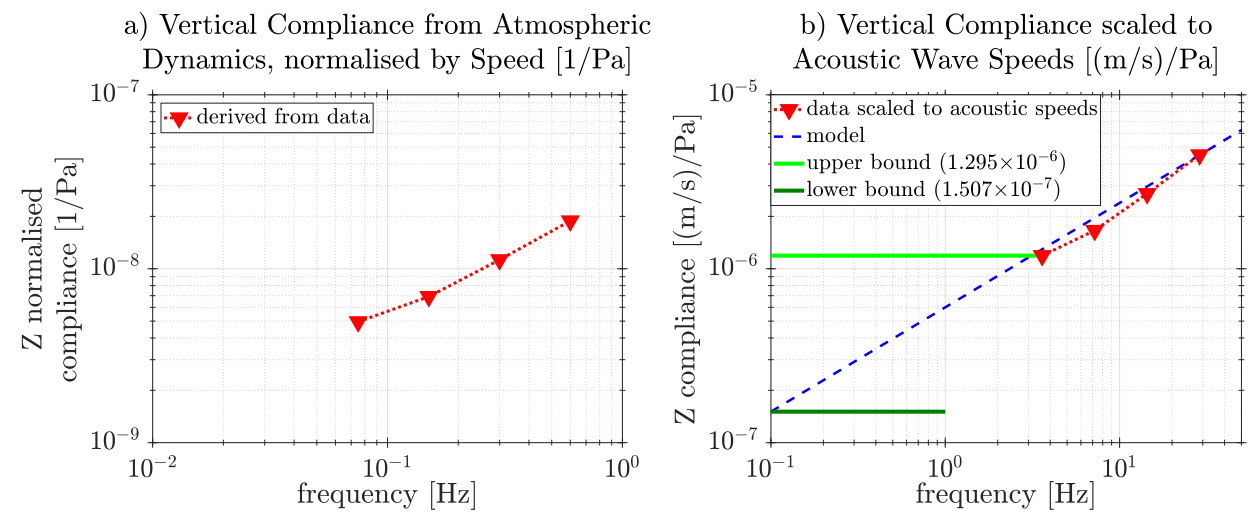

Figure 2. a) Average values of vertical compliance of atmospheric pressure perturbations normalized to wind speed (in $1 / \mathrm{Pa}$ ) as a function of frequency (in $\mathrm{Hz}$ ), estimated through compliance measurements using the VEL outputs of SEIS-VBB sensors (Garcia et al., 2020). b) Vertical compliance (in $(\mathrm{m} / \mathrm{s}) / \mathrm{Pa}$ ) of acoustic waves as a function of frequency (in $\mathrm{Hz}$ ) by conversion of vertical compliance from atmospheric pressure perturbation to acoustic waves through Eq. (11). The blue dashed line represents the empirical model of acoustic compliance extended in the direction of lower frequencies with a power law frequency dependence in the $0.1-1 \mathrm{~Hz}$ range. The dark (resp. light) green solid line represents the expected lower (resp. upper) bound for vertical compliance: $1.507 \times 10^{-7}(\mathrm{~m} / \mathrm{s}) / \mathrm{Pa}\left(\right.$ resp. $\left.1.295 \times 10^{-6}(\mathrm{~m} / \mathrm{s}) / \mathrm{Pa}\right)$.

\section{Wave Propagation Simulation Tools}

This Section presents the simulation tools used to model the propagation of acoustic waves in the Martian atmosphere, as well as the subsequent synthetic ground pressure perturbations and ground movements.

\subsection{Ray Tracing Modeling Tool: WASP-3D}

Ray tracing simulations are performed using WASP-3D, implementing the propagation of acoustic waves in a stratified, moving medium (Dessa et al., 2005). It can operate in three-dimensional spherical coordinates though for comparison with fullwave simulations described later, acoustic rays are traced in Cartesian coordinates. Conventional acoustic ray theory relies on the high-frequency approximation and is only exact in the geometric limit and does not allow modeling of the conversion to ground motion. However, this numerical approach is computationally very efficient, and thus particularly useful to grasp the overall propagation pattern in a variety of atmospheric configurations. Namely, WASP-3D primary outputs are travel times and ray trajectories. These simulated parameters allow a straightforward interpretation of the recorded acoustic phases. 


\subsection{Full Wave Numerical Modeling Tool: SPECFEM-DG}

The simulation tool SPECFEM-DG extends the widely-used SPECFEM software that employs a spectral element method (Komatitsch \& Vilotte, 1998; Komatitsch \& Tromp, 1999; Tromp et al., 2008) to model seismic wave propagation. SPECFEM stands for SPECtral Finite Elements Method, and the DG extension stands for Discontinuous Galerkin. The main evolution introduced in SPECFEM-DG is the coupling to the full system of Navier-Stokes equations for atmospheric media, as detailed by Brissaud et al. (2017). Note that this approach includes non-linearities, $\mathrm{CO}_{2}$ attenuation, and horizontal wind (and vertical variations of that wind, which are crucial for wave refraction).

The coupling at the fluid/solid boundary is done in two directions. Fluid-tosolid coupling is done by enforcing continuity of the normal stress and normal velocity. Solid-to-fluid coupling is done following the method described by Terrana et al. (2018). Outer boundary conditions are chosen periodic on left/right boundaries, and absorbing on the bottom (solid) and top (fluid) boundaries.

The numerical method relies on weak formulations through a spectral element method, continuous for the elastic domain (Komatitsch \& Vilotte, 1998; Komatitsch \& Tromp, 1999; Tromp et al., 2008), and discontinuous for the fluid domain (Brissaud et al., 2017). Time integration is explicit (optimal five-step fourth-order Runge-Kutta scheme (Carpenter \& Kennedy, 1994)).

SPECFEM-DG can intrinsically model waves in all frequency bands, from lowfrequency gravity waves to the infrasonic band and up to high-frequency acoustic modes (Brissaud et al., 2017; Spiga et al., 2018). The mechanical coupling enables both wave-wave transmission (Brissaud et al., 2017; Martire et al., 2018) and ground elastic deformations described here as compliance effects (see Appendix A for a validation). The outputs of this software are full wavefields. Consequently, one can extract, in particular, simulated seismograms (of, e.g., ground velocity $V_{x, z}(t)$ ) and simulated absolute pressure records (including pressure perturbations $\Delta P(t)$ ).

\section{Numerical Simulations of Acoustic Waves in Martian Dusk Condi- tions}

\subsection{Models}

The atmospheric models used are all generated from the Mars Climate Database (MCD) for the InSight landing site and the season of InSight landing (Forget et al., 1999; Millour et al., 2018). They are regularized and smoothed using splines to avoid the creation of spurious non-physical signals at first-order discontinuities.

Sub-surface models used are also stratified and based on pre-landing studies (Delage et al., 2017; Morgan et al., 2018). Though the results of this paper could be straightforwardly updated using the most recent inversion results, we choose for the sake of simplicity to keep only one sub-surface model. It is plotted in terms of Young's modulus and Poisson ratio in Figure 1 (as the "3-layers model"), or in terms of seismic velocities in Figure A3.

\subsection{Full-Wave SPECFEM-DG Simulations}

A full-wave 2D simulation was performed to highlight the key features of the infrasonic waves in a typical Martian dusk atmosphere. The atmospheric MCD model is chosen at InSight's sol 0, $20 \mathrm{~h} \mathrm{LMST}$, at the InSight landing site. The 2D plane is chosen along azimuth $160^{\circ}$, meaning the $x$-axis is positive towards South-Southeast. The model is plotted in Supplementary Figure S1. It is chosen range-independent because of the assumptions taken by SPECFEM-DG (Brissaud et al., 2017); this choice is assumed to hold for acoustic propagation under $300 \mathrm{~km}$ range on Mars. 
The chosen source is a broad spectrum Gaussian atmospheric excitation:

$$
S(t, x)=A \exp \left(-\left(\pi f_{0} t\right)^{2}-\frac{\left\|x-x_{0}\right\|_{2}^{2}}{\sigma^{2}}\right),
$$

with $A$ a multiplying factor (having no real importance as long as the waves remain linear), $f_{0}=3 \mathrm{~Hz}$ the dominant frequency, $\sigma=30 \mathrm{~m}$ the spatial spreading, and $x_{0}=(0,830) \mathrm{m}$ the source localization in $(x, z)$ coordinates. The source's altitude is chosen in order to input its energy to the lower atmospheric levels, and therefore to better excite the nocturnal surface waveguide. The waveform and Fourier transform of this source are plotted in Supplementary Figure S6. This source term is inserted in the energy equation of the Navier-Stokes system.

The ground/atmosphere boundary is flat (no topography) and located at $z=0$. The numerical domain spans from a depth of $5 \mathrm{~km}$ to an altitude of $30 \mathrm{~km}$. The horizontal span of the computational domain is designed with periodic boundary conditions enabling horizontal propagation up to $200 \mathrm{~km}$ in each direction from the source.

Recording stations are spread along the interface, sampling either ground pressure perturbations $\Delta P$ or ground movements $V_{x, z}$.

Using this simulation, we retrieve that the atmospheric refraction of acoustic waves is favored by the two types of atmospheric waveguides (introduced in Section 2.1), yielding distinctive pressure and velocity signatures at ground level.

As shown in Figure 3, this impulsive atmospheric excitation generates in the tropospheric waveguide short wave trains due to the reflection/refraction process, appearing over long time intervals. In this particular simulation, we retrieve short $(<10 \mathrm{~s})$ wave trains appearing over a $10 \mathrm{~s}$ time interval at horizontal distance $\left|x-x_{0}\right|=40 \mathrm{~km}$, over $50 \mathrm{~s}$ at $80 \mathrm{~km}$, or over $75 \mathrm{~s}$ at $120 \mathrm{~km}$.

Acoustic waves in the nocturnal waveguide become trapped, i.e., are reflected on the ground and refracted at the wind jets. Because this duct is shallow, constructive interference causes the guided waves to be subject to dispersion (Negraru \& Herrin, 2009). The impulsive source pulse translates into longer, dispersed, wave trains. In this simulation, we retrieve a single wave train with a duration of about $10 \mathrm{~s}$ at $\left|x-x_{0}\right|=40 \mathrm{~km}, 15 \mathrm{~s}$ at $80 \mathrm{~km}, 25 \mathrm{~s}$ at $160 \mathrm{~km}$, or $35 \mathrm{~s}$ at $180 \mathrm{~km}$.

Figure 3 highlights these features on synthetics. The trapped waves have a phase speed $v_{\phi} \in[245,259] \mathrm{m} / \mathrm{s}$, corresponding to the range of effective sound speed undergone in the nocturnal waveguide (Supplementary Figure S1). This phenomenon agrees with the "surface mode" found in similar simulations with terrestrial atmospheric models (Waxler et al., 2008).

A key feature of the narrow waveguide is that infrasound propagating in it appears to be dispersed. Along the passage of the wave train, the frequency contents appear to glide from low frequencies (about $0.2 \mathrm{~Hz}$, traveling faster) to high frequencies (about $1 \mathrm{~Hz}$, traveling slower). This effect can be seen in Figure 3, and is detailed in Supplementary Figure S7. Furthermore, this dispersion curve agrees with the narrow duct model developed by Negraru and Herrin (2009).

We also remark that the wavetrain is contained in an envelope of varying amplitude (Figure 3, Supplementary Figure S7). Because of the dispersive characteristic, the maximum amplitude of the envelope corresponds to a single frequency (of about $0.4 \mathrm{~Hz}$ in this case). Consequently, the spectrum of the guided waves can appear to be monotone (Figure 3, Supplementary Figure S9), though in reality it is a fully dispersive phenomenon.

Furthermore, note that this simulation can be seen as the impulsive response of the considered atmospheric model. As such, one can extrapolate these impulsive results by convolving the impulsive source by a long-duration source. Consequently, we believe that similar wave trains can be expected to have a much longer duration, only necessitating an excitation over a longer duration or wave scattering due to the 

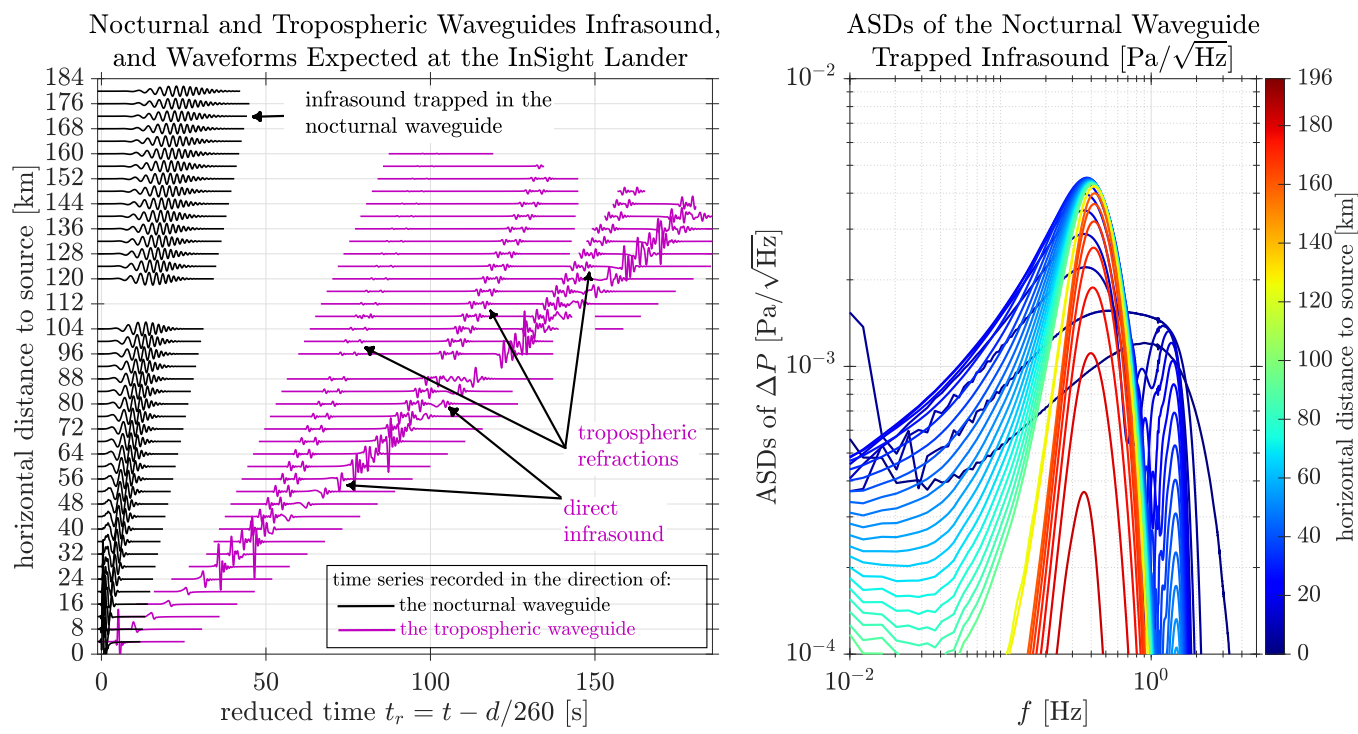

Figure 3. Synthetics of the full-wave simulation discussed in Section 4.2. Left: time-distance plots of synthetic ground pressure perturbations, shown in two directions on the same plot. In purple, synthetic time series in the tropospheric waveguide direction; and in black, in the nocturnal waveguide direction. Time is the same reduced time for both sets of curves. Distance is absolute horizontal distance to source (in either direction). All amplitudes were normalized in order to better highlight the arrivals. Right: Amplitude Spectral Densities (ASDs) of the synthetic wave trains in the nocturnal waveguide, as function of horizontal distance to source. The monotone excitation of this waveguide is clear, as close as $\simeq 40 \mathrm{~km}$ from the source (light blue shade). The dispersion of those waves is also clear between 0.2 and $1 \mathrm{~Hz}$, as also depicted in the dedicated Supplementary Figure S7.

heterogeneities of the waveguide. This in turn could explain the long duration signals observed by SEIS, presented later in this paper.

As mentioned in the introduction, similar infrasonic wave trains have been recorded on Earth, and linked to surface acoustic waveguides (Herrin et al., 2006; Negraru \& Herrin, 2009).

Panel a) of Figure 4 presents the relative amplitude of the two types of phase, either refracted by tropospheric winds or trapped in the nocturnal waveguide. At close distances from the source, the amplitudes of both signals are comparable. However, in the far-field, the two types of waves exhibit distinctive amplitude decreases. The energy of the tropospherically-refracted waves is quickly split in the refraction process. Furthermore, since they travel longer and higher in the atmosphere, they will undergo higher attenuation (Supplementary Figure S1). Whereas, the guided waves in the nocturnal waveguide see their amplitude decrease far less quickly. In the far-field, the trapped waves' amplitude is expected to be higher than that of the refracted waves. Infrasound in the nocturnal waveguide see their amplitude decrease $\propto r^{-1 / 2}(r$ the horizontal distance to the source), while the waves in the tropospheric waveguide undergo a decrease $\propto r^{-3 / 2}$.

As described in panel b) of Figure 4, under each type of infrasound (either guided or refracted), the $V_{z} / V_{h}$ ratio of vertical to horizontal ground velocity appears to remain $\geq 2$. This behavior may occur due to seismic effects only (without any infrasonic wave) but requires to have a very specific soil composition and structure. As introduced 
using the compliance theory, infrasonic waves propagating horizontally are expected to produce such ratio of vertical to horizontal ground motion (Figure 1).

The polarization angle of ground movements is presented in panel c) of Figure 4. In simulation, we retrieve that the ground polarization is nearly always vertical $\left(0^{\circ}\right)$. This is expected since the energy coming from a source at such a low altitude $\left(z_{0}=830 \mathrm{~m}\right)$ is always encountering the ground at large, horizontal, incidences. At caustics (e.g., at $40 \mathrm{~km}$ horizontal distance), the acoustic wave due to some tropospheric refraction encounters the ground at low incidence angles, since it arrives from nearly directly above, and we observe a shift to high ground polarizations. The sign of the ground polarization angle is also linked to the direction of propagation of the waves. Thanks to this effect, one may be able to infer the direction of arrival of ground-coupled acoustic waves on InSight's data.

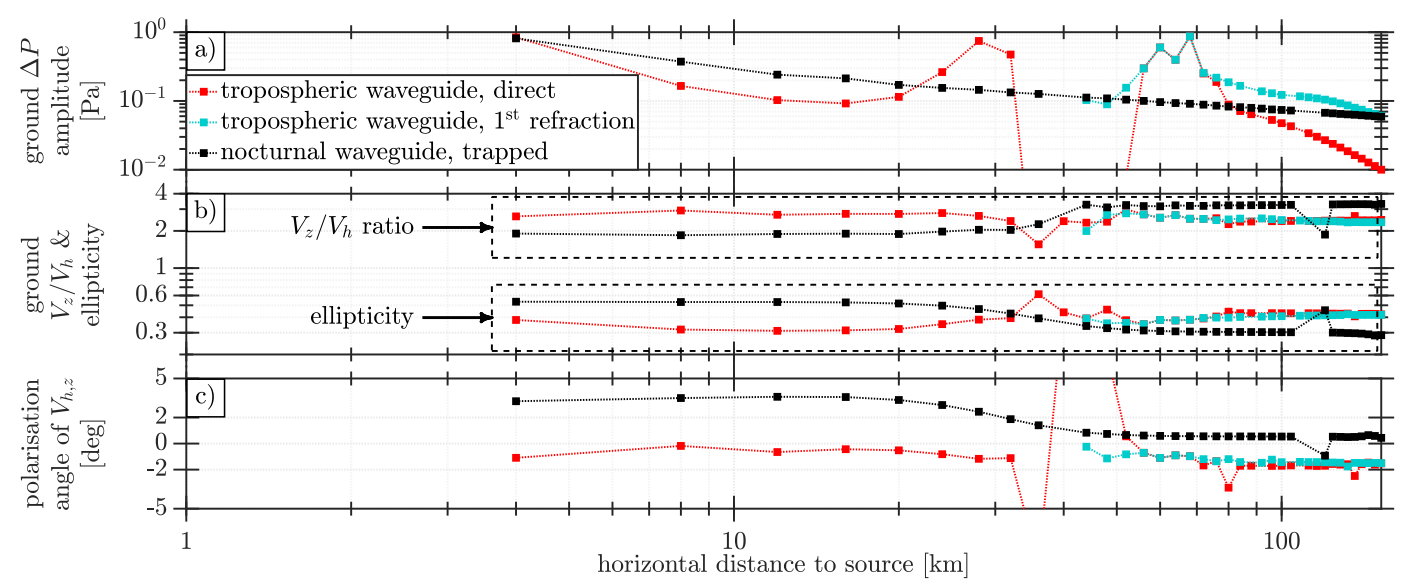

Figure 4. Characterization of ground-recorded pressure perturbation $\Delta P$ and subsequent ground movements $V_{h, z}$, from synthetics. On each panel, quantities are plotted for each of three different types of atmospheric waves: the direct wave in the tropospheric duct direction (red), the first tropospherically-refracted wave (blue), and the guided/trapped wave in the surface duct (black). a) Amplitude of the normalized ground pressure perturbation $\Delta P$ synthetics. b) From the $V_{h, z}$ synthetics, $V_{z} / V_{h}$ ratio (values $>1$ ) and ellipticity (values $<1$ ). c) Polarization angle of the $V_{h, z}$ synthetics, i.e., the angle of the semi-major axis of the ellipse traced by $\left(V_{h}(t), V_{z}(t)\right)$. Since the simulation is conducted with the $x$-axis pointing towards azimuth $160^{\circ}$, a polarization angle $>0^{\circ}$ means the polarization is slightly tilted towards South-Southeast. This is namely the case for the guided waves, which propagate towards South-Southeast. Recall that the ground movements $\left(V_{z, h}\right.$, see panels b) and c)) are here directly due to the pressure perturbation above ground $(\Delta P$, see panel a)).

Additional full-wave simulations were performed to study the sensitivity of the nocturnal waveguide's frequency response, and understand the key parameters. They are summarized in the next Sections (4.2.1, 4.2.2, 4.2.3, and 4.2.4).

\subsubsection{Sensitivity to the Atmospheric Model}

The MCD software (Forget et al., 1999; Millour et al., 2018) offers variability following realistic scenarios, aside from the standard model, for generating Martian atmospheric models. For example, the effects of solar wind can be explored, as well as dust conditions, or relative atmospheric temperature. In short, a certain degree of 
variability can be taken for the models' generation, in order to account for the actual variability of the Martian atmosphere.

Using various MCD models, we established that the nocturnal waveguide's frequency response is closely linked to the altitude of the wind jet. This sensitivity is illustrated in Supplementary Figure S2.

The key point here is that changes in atmospheric conditions may change the shape of the acoustic ducting zones. In turn, changing the nocturnal waveguide's shape causes the main excited frequency to shift, but also the whole dispersion response to vary (Supplementary Figure S2).

We expect to be able to infer the nocturnal waveguide's shape by 1) studying the dispersion curve of trapped infrasound events (Herrin et al., 2006; Negraru \& Herrin, 2009), and 2) making use of the APSS (Spiga et al., 2018; Banfield et al., 2019, 2020) to probe atmospheric specifications at ground level. However, we will see in Section 4.2.2 that the ground model also plays a role.

\subsubsection{Sensitivity to the Ground Model}

Figure 5 presents the sensitivity to the ground model. The pre-landing ground model, layered and with some soft regolith on top, plays the role of a high-pass filter on ground velocity, consequently enhancing further the monotone aspect of trapped waves.

Aside from the high-pass effect, different ground structures seem not to influence the overall fact that guided atmospheric waves produce a dispersive pattern peaking at one frequency. However, a soft soil appears to enhance the resonance, while on hard soil the infrasound decays more quickly.

\subsubsection{Sensitivity to Atmospheric Attenuation}

In order to examine the effects of atmospheric attenuation, we performed some other full-wave simulations without any attenuation. We set $\mu=\kappa=0$ ( $\mu$ the dynamic viscosity, $\kappa$ the thermal conductivity), causing $\alpha_{\mathrm{cl}}=\alpha_{\text {rot }}=0$ i.e., neither classical nor rotational attenuation. We also force $\alpha_{\mathrm{vib}}=0$ (no vibrational relaxation). We found that attenuation does not play a major role in the $0.1-1 \mathrm{~Hz}$ excitation, which was expected since the attenuation effect is only truly important above $\simeq 2 \mathrm{~Hz}$.

Higher-order dispersive modes may exist for some duct shapes (Negraru \& Herrin, 2009). However, they are expected to decay quickly due to the highly attenuative Martian atmosphere. From additional full-wave simulations, we found that the atmospheric model plotted in Supplementary Figure S1 could not support higherorder modes (even after removing attenuation). Some waveguide shapes were found to support a higher-order mode (just above $1 \mathrm{~Hz}$ ), but it was drastically attenuated (Supplementary Figure S2).

\subsubsection{Sensitivity to the Source's Altitude}

We also investigated the effect of source altitude by checking outside, near, and within the nocturnal waveguide. As expected, exciting directly the wave duct from within is more efficient, in the sense that a monotone frequency is then properly enhanced. With higher sources, the monotone effect is still present at large distances, but 1) it is not enhanced as much and 2) the dispersive mode first appears at a greater range.

This is expected since impinging waves need to be coming from large incidences in order to post-critically reflect on the wind jet and create the interference pattern (Negraru \& Herrin, 2009). Consequently, with higher sources, the dispersive mode cannot develop as efficiently. 

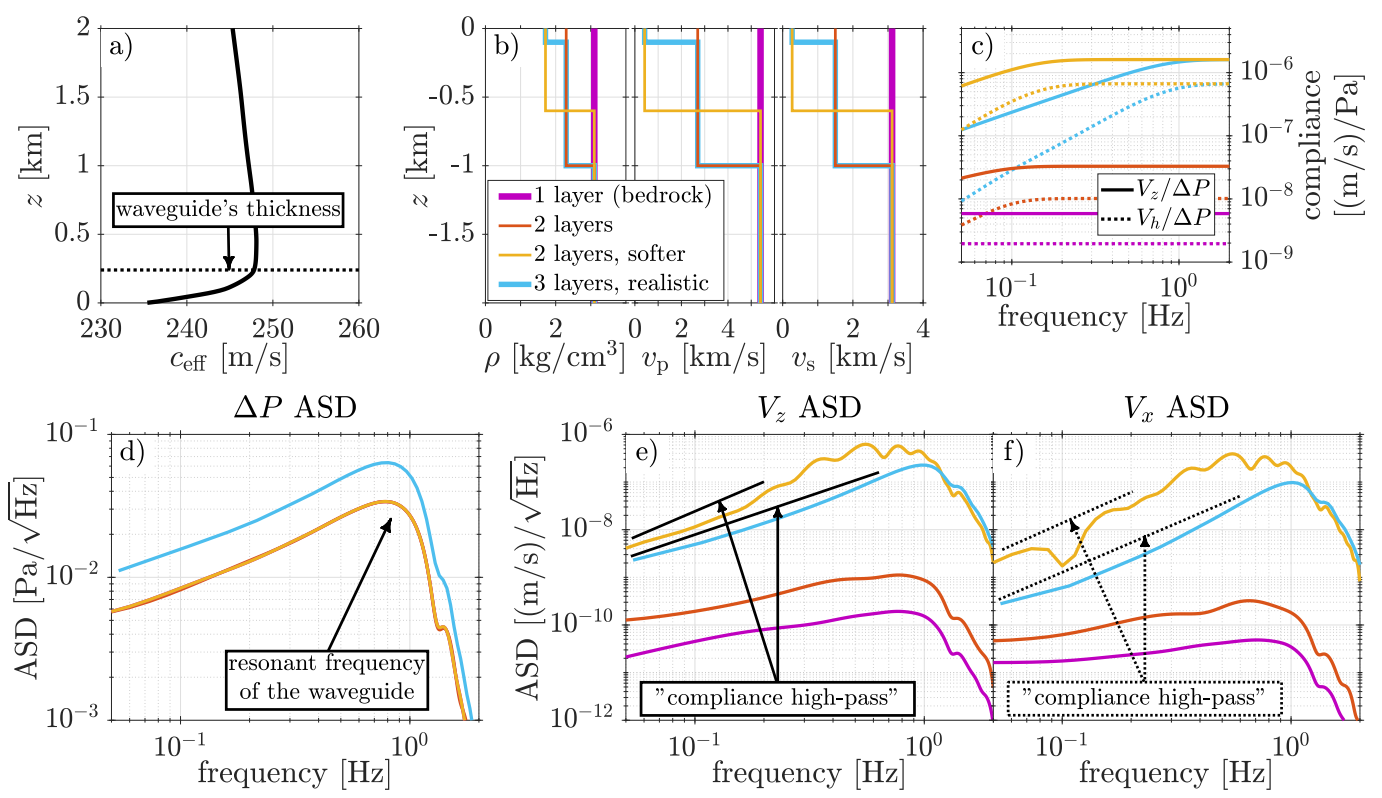

Figure 5. Sensitivity of the trapped infrasound's frequency response to the ground model.

a) Effective sound speed for the atmospheric model used (extracted from MCD for sol 189, 19h LMST, along azimuth $170^{\circ}$ ). b) Seismic parameters for the tested ground models. The colors used here and in the next sub-plots encode each model. c) Compliance coefficients for each ground model (Kenda et al., 2017, 2020), as function of frequency, and for a propagation speed of $235 \mathrm{~m} / \mathrm{s}$ (effective sound speed at the ground, see panel a)). Bottom (panels d)-f)): Amplitude Spectral Densities (ASDs) of synthetics, recorded at altitude $z=0$ and an horizontal distance of $r=32 \mathrm{~km}$ from source, for each ground model. d) Ground pressure perturbations $(\Delta P)$. The 1layer and both 2-layers ground models are on top of each other. e) Ground vertical velocity $\left(V_{z}\right)$, and f) ground horizontal velocity $\left(V_{x}\right)$. On those $V_{x, z}$ ASD plots, the "high-pass" effect stemming from compliance conversion are highlighted by the black lines. Note that the $\Delta P$ ASD spans 2 decades, whereas the $V_{z, x}$ one spans 6 . See Supplementary Figure S2 for a study of the sensitivity to the atmospheric model.

\subsubsection{Summary of Results from Full-Wave Simulations}

Infrasonic waves can be refracted from the tropospheric waveguide, translating into short wave trains over long periods (Figure 3). Infrasound may also become trapped in the nocturnal waveguide, yielding a single long wave train, and propagation there is subject to the minimum amplitude decay (Figure 3). This trapped infrasonic wavetrain is subject to dispersion, with a center frequency depending chiefly on the altitude of the dusk low-level wind jet. A source below this jet (i.e., within the waveguide) is most efficient in exciting the guided dispersive mode.

Propagation over a layered and relatively soft soil (such as the one presented in Figures 1 and A3) is enhancing the monotone effect (Figure 5). Indeed, because of its incident angle, guided infrasound falls into the compliance conversion mode (Section 2). Layered sub-surfaces act as a high-pass filter in the conversion from pressure perturbations to ground velocity (Figure 5). For both infrasound in the nocturnal waveguide and refracted by the tropospheric jet, the ratio $V_{z} / V_{h} \geq 2$. For the guided infrasound following the compliance theory, $V_{z}$ has a $-90^{\circ}$ phase shift relative to $V_{h}$ (Section 2.3.2). 


\subsection{Ray Tracing Simulations}

Acoustic ray tracing simulations are performed using the same atmospheric model described above (Supplementary Figure S1). The source's altitude is also set to $z_{0}=$ $830 \mathrm{~m}$. The results presented in Figure 6 highlight very distinctive features in the two directions.
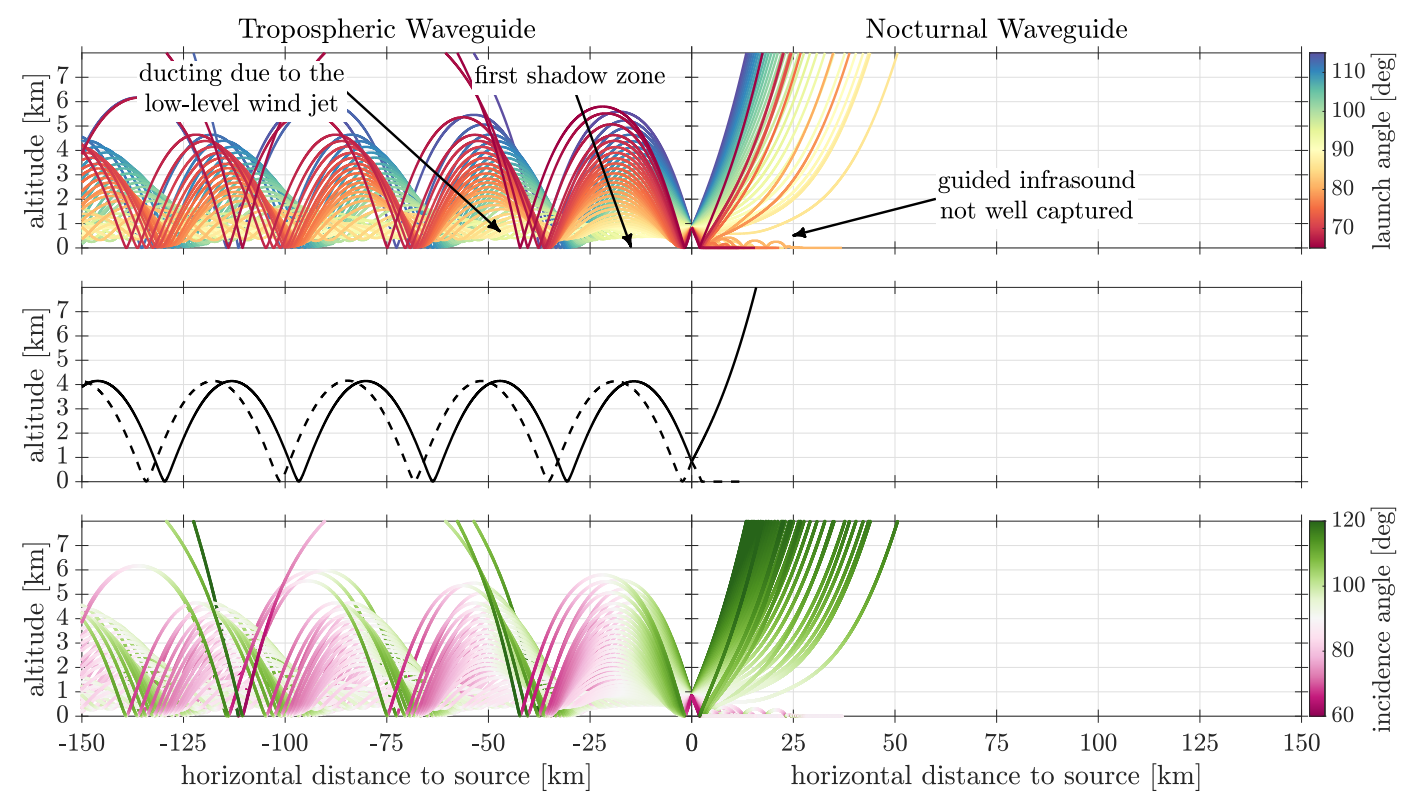

Figure 6. Acoustic rays propagation in the tropospheric and nocturnal waveguides, on the left and right panels respectively. Top: rays color-coded with launch angle. Middle: ray trajectories for a couple of launch angles $\left(70^{\circ}\right.$ as dashed line, and $110^{\circ}$ as plain line). Bottom: rays colorcoded with incidence angle with respect to the direction of propagation. Rays are traced using the reference background model (sol 0, 20h LMST, landing site location, plotted in Supplementary Figure S1).

In the tropospheric waveguide direction, no rays are reaching the ground up to a certain range. This region is called an acoustic "shadow zone", a feature of infrasound propagation frequently observed on Earth (de Groot-Hedlin, 2017). During nighttime on Mars, the shadow zone has a finite length due to the presence of large-amplitude tropospheric winds (Garcia et al., 2017). The end of the shadow zone is marked by a jump in the density of rays guided at low altitudes, translating into an amplitude increase in the full-wave simulation. This feature repeats as rays bounce on the ground at regular intervals. We note that each tropospheric refraction signature is composed of two successive impulses that appear when the acoustic source is located above the ground. This is because one impulse stems from the up-going wave, and the other from the down-going wave reflected off of the ground. From ray tracing simulations we expect an increase of the time delay with the source height; and expect only one phase for a source on the ground. Rays with larger launch angles sound the troposphere higher and reach larger distances. A decrease of the density of rays combined to longer propagation paths explain the rapidly decreasing amplitude with distance described above with the full-wave simulations.

The rays guided at very low altitude $(\leq 500 \mathrm{~m})$ confirm the acoustic wave trapping phenomenon in the nocturnal waveguide. Note that in our acoustic ray simulation, 
rays propagate at limited range $(\leq 25 \mathrm{~km})$ while the full-wave simulation succeeds to capture resonant waveforms at long ranges.

These arguments obviously only hold for the studied season (sols 0-189). Any change in the latter can modify the preferential ducting direction. That being said, the wind direction remains the most crucial constraint.

\subsection{Discussion}

Two significant differences between the ray tracing and the full-wave simulations need to be discussed.

First, a shadow zone can be distinguished in the ray simulation (Figure 6), while the full-wave simulation captures signals continuously (Figures 3 and 4). In fact, the full-wave synthetic signals recorded at distances corresponding to the rays' shadow zone (e.g., 5-30 km) are direct wavefronts emanating from the source. The results of the ray simulation show that the launch angles expected to reach the shadow zone are in fact ducted and neither touch the surface nor escape the waveguide. This makes sense with respect to the increase of effective sound speed with altitude close to the ground in the tropospheric waveguide direction, corresponding to the nocturnal waveguide in the other direction (Supplementary Figure S1).

Secondly, in the nocturnal waveguide, rays quickly escape to higher altitudes, while they are well-captured in the full-wave simulations.

Both differences are nothing but a direct consequence of the geometric hypothesis used in the rays' approach. Since the wavelengths at play are comparable to the nocturnal waveguide's thickness, the rays cannot capture well the phenomena at all launch angles.

\section{Martian Infrasound Interpretation of some monotone Seismic Events}

SEIS recorded during the first 300 sols of the InSight mission a few events that are sharing the following common features: monotone energy arrival, larger vertical than horizontal velocity signal, sometimes clear polarization of energy arrivals, and no clear seismic phases (i.e., $\mathrm{P}$ - and S-waves arrivals).

This Section aims to apply the results obtained through our numerical simulation to InSight's SEIS' data, and namely to provide a new interpretation based on infrasound guided in the nocturnal waveguide. First, we select and analyze some SEIS events. Then, we provide a discussion on the potential source of infrasound causing those.

\subsection{Analysis of Selected SEIS Events}

We reviewed every low-frequency and broadband SEIS events detected by the Marsquake Service (MQS) between sol 0 and 299 (InSight Marsquake Service, 2020), searching for the key features highlighted above. First, the event is deemed monotone if the Amplitude Spectral Densities (ASD) of the vertical and horizontal components exhibit a narrow-band peak sticking out of the noise level in the $0.1-1 \mathrm{~Hz}$ range. Using the very same ASDs, we select those having a peak larger on the vertical component than on the horizontal ones. Studying the time series, we can exclude those having clear seismic phases (clear P- and S-wave arrivals), and we can explicitly choose those having coherent signals between SEIS' channels and a $\pm 90^{\circ}$ phase shift between the horizontal and vertical channels. Finally, from the full-wave numerical simulations and compliance theory, specific phase shifts between the $\Delta P$ and $V_{z, h}$ records are expected for an infrasonic interpretation (Section 2.3.2). We restrict our search to events presenting these features. Supplementary Figure S3 shows the ASD analysis 
Table 1. List of selected SEIS events suspected to be caused by infrasound trapped in the Martian nocturnal surface waveguide. The event name encodes the InSight sol during which it occurred. The start times are fetched from the MQS catalog (InSight Marsquake Service, 2020). The sunset times are computed using the pyMarsTime package (https://github.com/eelsirhc/ pyMarsTime, implementing the algorithm by Allison (1997) and Allison and McEwen (2000)). LMST stands for Local Mean Solar Time.

\begin{tabular}{c|c|c}
\hline Event & Start Time (hh:mm:ss LMST) & Local Sunset (hh:mm:ss LMST) \\
\hline S0105a & $18: 06: 05$ & $18: 44: 03$ \\
S0133a & $20: 44: 23$ & $18: 37: 05$ \\
S0152a & $21: 26: 16$ & $18: 31: 34$ \\
S0185a & $18: 28: 26$ & $18: 21: 29$ \\
S0189a & $19: 15: 27$ & $18: 20: 17$ \\
S0234c & $20: 45: 45$ & $18: 08: 02$ \\
S0234d & $22: 03: 28$ & $18: 08: 02$ \\
S0290b & $22: 11: 17$ & $17: 57: 03$ \\
\hline
\end{tabular}

(i.e., the peaks' central frequencies, widths, and amplitudes) of all low-frequency and broadband SEIS events before selection.

Following this review process, we selected eight events, listed in Table 1. Those events share the same additional features: their central frequency varies from sol to sol, and they are mainly seen right after local dusk (Table 1). Figure 7 presents the ASD analysis of those events (peaks' central frequencies, widths, and amplitudes).

A seismic interpretation of those events is difficult to sustain. First, because Martian seismic events are not producing mono-frequency signals (see (Giardini et al., 2020)'s Figure 4). Secondly, if a resonance was truly generated by the sub-surface, different seismic events recorded at the same station could not have a varying frequency. Thirdly, no clear seismic phases (of P- and S-wave arrivals) can be identified.

We thus consider an explanation from the point of view of infrasound pressure fluctuations, causing non-negligible ground motion measured by SEIS. Most notably, the fact that those events occur at dusk concurs with the appearance of the nighttime low-level jet, and is thus particularly relevant to the dispersive features highlighted by the full-wave simulations presented in Section 4.2. The variability of the atmosphere alone (Section 4.2.1, Supplementary Figure S2) can explain the variability in frequency. The high-pass effect on ground velocity (Section 4.2.2) can be responsible for the fact that none of these events seems to occur below $0.3 \mathrm{~Hz}$.

Among all the selected events, we found two for which our acoustic interpretation is fully compatible with SEIS' data: S0133a and S0189a. In the remaining of this Section, we focus on S0189a. We suspect that the other events are also due to trapped infrasound, however noisier data makes our demonstration less robust. That being said, the exact same rationale can be applied to all events.

S0189a happened on InSight sol 189 at 19:15 LMST (on the $9^{\text {th }}$ of June 2019 at 05:40 UTC). For this event, the TWINS (Temperature and WINd Sensor) instrument recorded a wind azimuth of $\simeq 100^{\circ}$ (almost due East) and a wind strength of $\simeq 2.3 \mathrm{~m} / \mathrm{s}$ on the lander.

Figure 7 presents the ASD analysis of S0189a, highlighting spectral energy spanning $0.5-0.9 \mathrm{~Hz}$ and a peak frequency of $f_{189 \mathrm{a}} \simeq 0.63 \mathrm{~Hz}$. Figure 8 presents the time series analysis of S0189a, based on the coherence $C_{\mathrm{VEL}_{\mathrm{Z}}, \mathrm{VEL}_{\mathrm{E}}}(f)$ between the vertical and horizontal (East) SEIS components. The coherence between some times series $x$ 

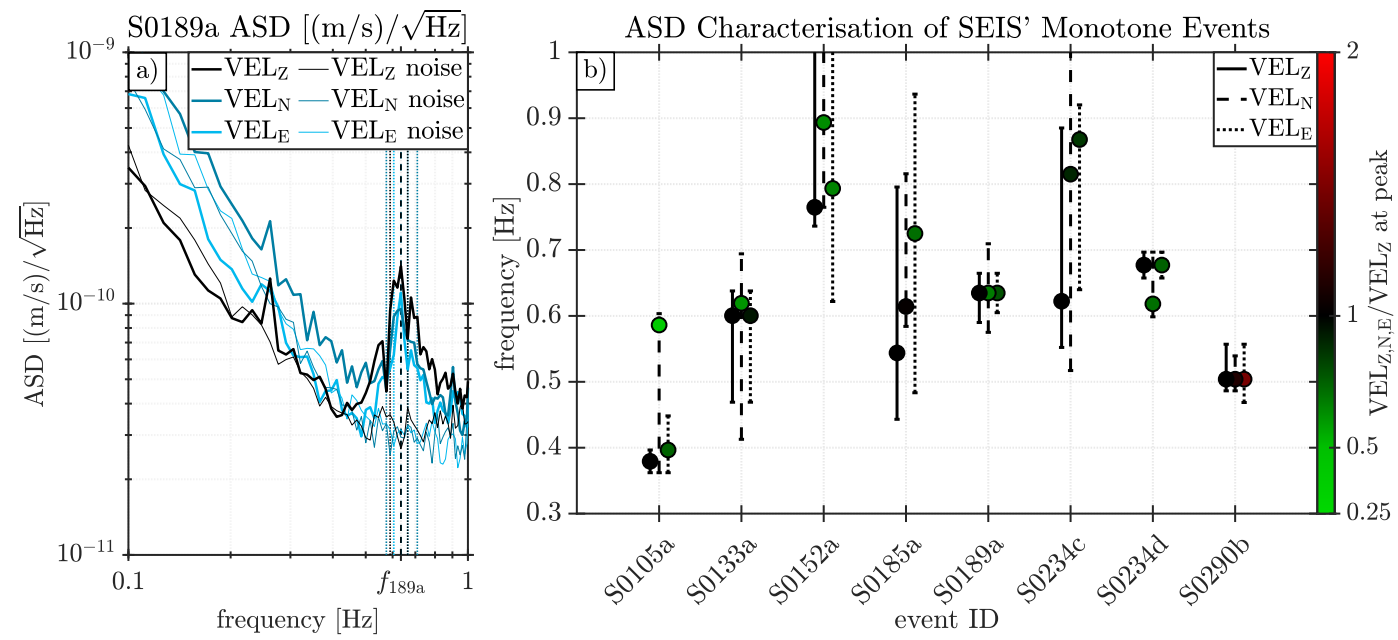

Figure 7. Characterization of monotone events using the peak in the Amplitude Spectral Densities (ASDs). a) Such ASD for the S0189a event. The central frequencies on each component are highlighted by the vertical dashed lines, and its half-height width by the dotted lines. b) For every selected event, the peak frequency (dot) and half-height width (bracket) obtained for each SEIS component (vertical Z as solid line, North $\mathrm{N}$ as dashed line, and East $\mathrm{E}$ as dotted line). The color of the dot represents the ratios $V_{\mathrm{Z}, \mathrm{N}, \mathrm{E}} / V_{Z}$ computed at the peak.

and $y$ is defined as

$$
C_{x y}(f)=\frac{\left|G_{x y}(f)\right|^{2}}{G_{x x}(f) G_{y y}(f)},
$$

with $f$ the frequency, and $G_{x y}(f)$ the cross-spectrum between $x$ and $y$. The coherence's phase of event S0189a exhibits a $90^{\circ}$ phase shift between SEIS' vertical and East components, which agrees with synthetics (Supplementary Figure S4) and compliance theory (Section 2.3.2). Hence, this also supports the guided infrasound interpretation.

Supplementary Figure S7 presents spectrograms aimed at comparing dispersion between our full-wave synthetics and S0189a's data. Dispersion in data is not clear, but we recall that we are very close to SEIS' noise level (Figure 7). Hence, we believe that the frequency peak seen in data is only the peak of energy in the middle of the dispersive spectrum's frequency range, as introduced in Section 4.2. The observed peak frequency $f_{189 a}$ agrees with realistic values obtained from synthetics (Supplementary Figure S7).

Supplementary Figure S5 presents the time series and spectral analysis of the S0133a event, for which the exact same conclusions can be made. The dispersion discussion of event S0189a also holds here. A spectrogram of S0133a is presented in Supplementary Figure S8, to be compared with Supplementary Figure S7's synthetic spectrogram (panels a) and c)).

In conclusion, we demonstrated that the monotone events recorded on SEIS are the conjunction of three effects. First, atmospheric pressure perturbations are low-pass-filtered due to $\mathrm{CO}_{2}$ relaxation, typically below $2 \mathrm{~Hz}$ (Bass \& Chambers, 2001; Williams, 2001; Petculescu \& Lueptow, 2007). Secondly, the narrow nocturnal waveguide appearing at dusk produces guided infrasound subject to dispersion in the 0.1-1 Hz range. This duct's frequency response varies (Supplementary Figure S2), particularly in terms of peak frequency, depending on its shape (Herrin et al., 2006; Negraru \& Herrin, 2009). Thirdly, the guided waves falling in the compliance conversion regime, a layered ground model acts as a high-pass filter for ground velocity (both 


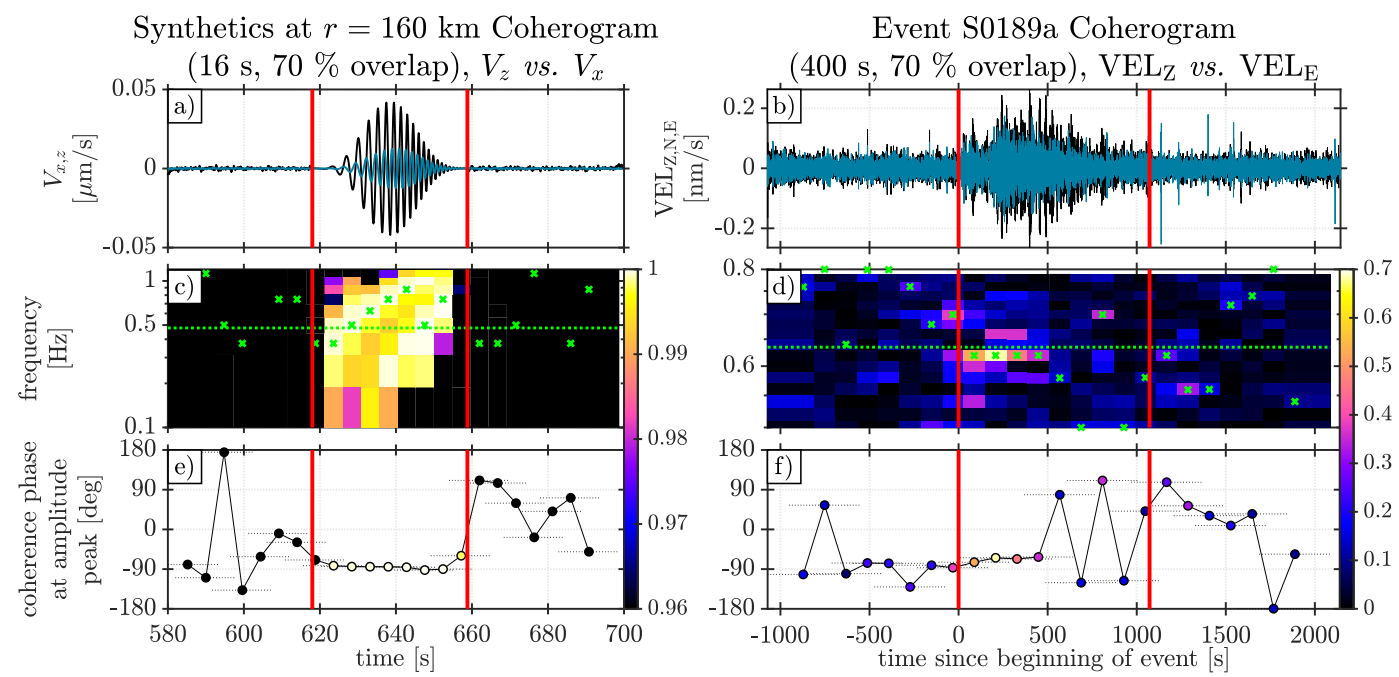

Figure 8. Coherence analysis. Left (panels a)-c)-e)): on synthetics, between $V_{z}$ (black) and $V_{x}$ (blue), for the simulation introduced in Section 4.2. Right (panels b)-d)-f)): on SEIS' data, between the vertical (black) and East (blue) components, for the S0189a event. Top (panels $\mathrm{a}$ and $\mathrm{b}$ ): band-pass filtered time series in the frequency range shown in panels (c) and (d), respectively. Middle (panels $\mathrm{c}$ and $\mathrm{d}$ ): coherence amplitude as function of time, with a rolling window (length $16 \mathrm{~s}$ in panel $\mathrm{c}$ and length $400 \mathrm{~s}$ in panel d) and $70 \%$ overlap. The value of the coherence amplitude is given by the color bars to the side of each panel. The peak amplitude in each time window is highlighted by a green cross. The peak frequency found in the Amplitude Spectral Densities is highlighted by the dotted green line. Bottom (panels e and f): coherence phase at the amplitude peak. The amplitude is recalled by the color of the points. On all panels, the event's start and end times are delimited by the red vertical lines. The band-pass filter is chosen around the peak frequency found in the event's ASD. The coherence is computed using the non-filtered data. Some white noise was added to the synthetics (panels a)-c)-e)) outside the "event" to prevent the coherence from being undefined (see Eq. (13)). A noisier version of the synthetics' coherence analysis is presented in Supplementary Figure S9.

horizontal and vertical), with cutoff typically around $0.4 \mathrm{~Hz}$ for pre-landing models (Figure 5).

Finally, we believe the lack of a clear dispersion pattern in these events' data only stems from the fact that they are close to SEIS' noise level; consequently, only the peak dispersion sticks out (Section 4.2, Supplementary Figure S7). Moreover, the compliance high-pass filtering is expected to enhance further the monotone aspect of ground motion records (Section 4.2.2). Future observations of higher-amplitude trapped infrasound on APSS will be necessary to confirm this point.

\subsection{Expected Infrasonic Signal on APSS' Pressure Sensor}

One could argue that no pressure perturbation was recorded by APSS' pressure sensor during the monotone SEIS events. However, using the compliance theory results (Section 2.3.2), we retrieve that no pressure perturbation could have been recorded for those events.

First, for perturbations moving at acoustic speed, we retrieve from data a lower bound of $[V / P]_{\min }=1.507 \times 10^{-7}(\mathrm{~m} / \mathrm{s}) / \mathrm{Pa}$ for the vertical compliance coefficients (Figure 2). The selected monotone events, when band-pass filtered in the $0.4-0.8 \mathrm{~Hz}$ 
range, all have a peak amplitude smaller than $V_{\max }=2 \times 10^{-10} \mathrm{~m} / \mathrm{s}$ (Figure 7, Figure 8 , Supplementary Figures S5, S7, S8). Consequently, the expected maximum pressure perturbation is:

$$
[\Delta P]_{\max }=\frac{V_{\max }}{[V / P]_{\min }}=\frac{2 \times 10^{-10}}{1.507 \times 10^{-7}}=1.3 \times 10^{-3} \mathrm{~Pa} .
$$

However, the noise level of APSS' pressure sensor in the $0.1-1 \mathrm{~Hz}$ range is $>2 \times 10^{-3} \mathrm{~Pa}$. Hence, the expected pressure perturbations for infrasound generating those SEIS events remain undetectable by the pressure sensor.

\subsection{Possible Infrasound Sources}

As demonstrated in Section 5.2, no pressure signal on APSS is expected for those events. A remaining element to sustain the infrasound interpretation is to uncover some atmospheric events that could act as a source.

Figure 9 shows pressure records around the events of interest. One can clearly distinguish gravity waves (GWs) appearing $1 \mathrm{~h}$ before and $1.5 \mathrm{~h}$ after the S0133a event. Some low-frequency activity below the Brunt-Väisälä frequency is present after the S0189a event, though there is no clear wave train in the time series.

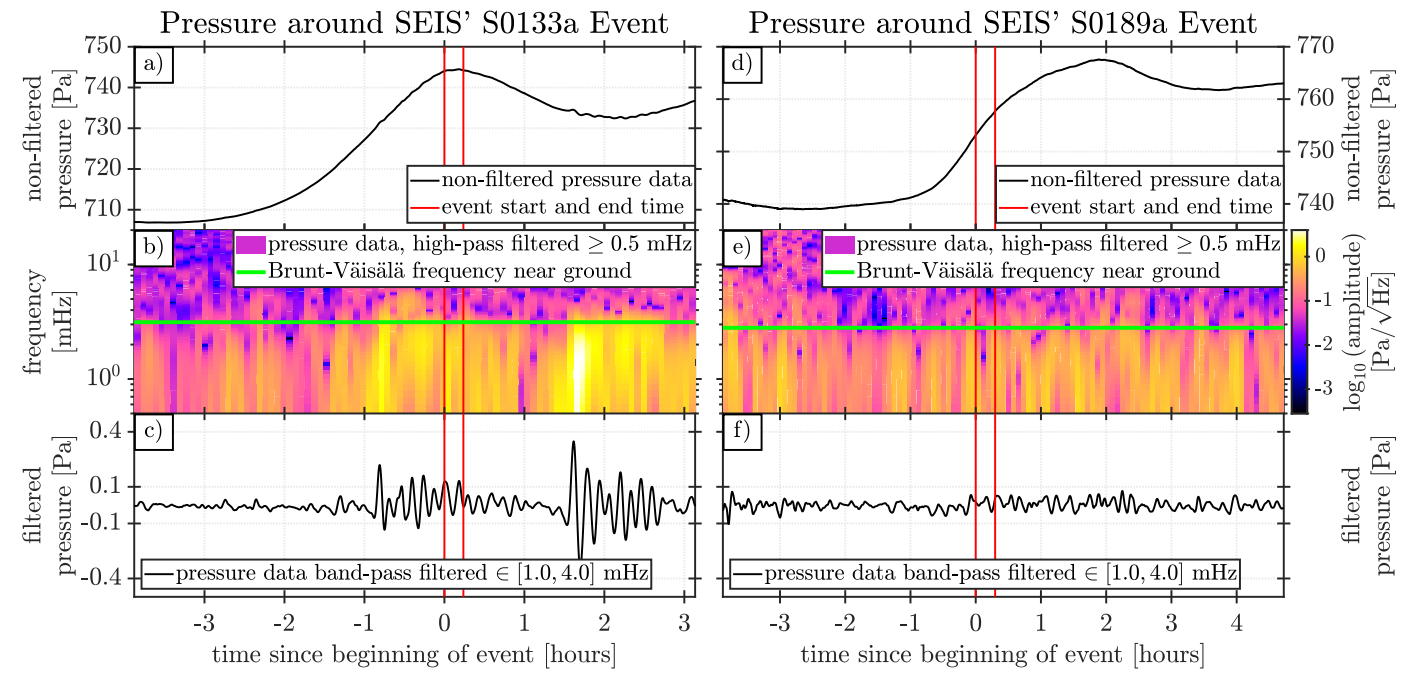

Figure 9. Pressure recorded by APSS around the S0133a (panels a)-b)-c)) and S0189a (panels d)-e)-f)) SEIS events. a) \& d): unfiltered time series. b) \& e): spectrograms (900 s window, $70 \%$ overlap) and Brunt-Väisälä frequency at the time of the event. The Brunt-Väisälä frequency highlighted in these panels is computed from the Mars Climate Database (MCD) models (Forget et al., 1999; Millour et al., 2018) at the corresponding dates and times. c) \& f): filtered time series in the gravity wave frequency band $(1-4 \mathrm{mHz})$.

The temporal coincidence of both an infrasound signal and gravity waves suggests a common source. Given the evening occurrence of those events, plausible sources are the following. Infrasound can stem from some wind/mountain interactions (e.g., Elysium Mons or the dichotomy boundary) in stable conditions (Campus \& Christie, 2010, Section 6.3.2). Wave emission can radiate from turbulent regions (Howe, 2002), and thus can be associated with either the collapse of the daytime planetary boundary layer or the transition from day to night (e.g., the arising of the nighttime low-level jet, which would then act as both a source and a guide for the gravity wave and 
infrasound). Finally, a source related to a dust storm is not to be excluded, but no major regional dust event was noticed at this moment of the InSight observations.

For a common source, since the horizontal phase speed of infrasound $\left(v_{\phi}^{\mathrm{i}}\right)$ is greater than that of gravity waves $\left(v_{\phi}^{\mathrm{g}}\right)$, infrasound may arrive first on the InSight lander, followed by the gravity wave train, which is one interpretation of these observations. Using the time delay, one only needs to determine the gravity wave train's phase speed $v_{\phi}^{\mathrm{g}}$ in order to get a source distance $\left(d=\Delta t /\left(1 / v_{\phi}^{\mathrm{g}}-1 / v_{\phi}^{\mathrm{i}}\right)\right)$. The backazimuth could be derived from the wind direction or the ground polarization angle recorded on SEIS (Section 4.2, Figure 4). However, precisely determining the phase speed of gravity waves using a single station remains very difficult. An estimate of the phase speed is possible when simultaneous oscillations of wind direction/speed are recorded along with oscillations of pressure (as is reported by Banfield et al. (2020)), but this was not the case for the events studied here.

Of course, some other sources unrelated to the atmosphere itself (impacts, meteor entries, etc.) could be involved, however as of today we lack the tools and data necessary to confirm such hypotheses. In that case, the gravity waves presented in Figure 9 would simply modulate the infrasound signal emitted by another source (unrelated to the gravity waves), contrary to the scenario explored in the previous paragraphs.

\section{Conclusion}

We performed numerical simulations of infrasound in the Martian coupled groundatmosphere system. We conducted full-wave simulations using SPECFEM-DG (Brissaud et al., 2017) that implements the mechanical coupling between the atmosphere and the ground, along with ray tracing simulations using WASP-3D (Dessa et al., 2005).

We predicted the pressure variations caused by the low-frequency acoustic (infrasonic) waves in the atmosphere, as well as the subsequent ground vibrations. We characterized the relationship between these two physical observables in terms of amplitude, frequency content, and phase shifts.

Most importantly, we characterized in Sections 2 and 4 how to discriminate between acoustic waves and wind advection using a single station. The pressure signal alone not being sufficient, one needs to analyze the ground motion. This characterization was then put in the scope of the on-going InSight mission. Additionally, in the future, the arguments developed in this contribution will allow the search for other infrasonic signals in SEIS/APSS data.

We then focused, in Section 5, on some events recorded by the seismometer SEIS. Because a seismic interpretation of these events is difficult to sustain, we studied them from the point of view of infrasound trapped in the nocturnal surface waveguide. Our findings altogether support the following interpretation: such events are the consequence of the trapped infrasound, and the product of a natural band-pass filtering phenomenon.

Firstly, the low-level wind jets appearing at dusk can trap acoustic waves in a nocturnal waveguide ( $\leq 2 \mathrm{~km}$ altitude, see Supplementary Figure S1). This narrow surface wind duct is prone to the propagation of a dispersive acoustic mode which frequencies lie between 0.1 and $1 \mathrm{~Hz}$ (Figure 3, Supplementary Figures S7 and S8). Secondly, the atmosphere filters out the high frequencies and any higher-order modes, because of the strong vibrational attenuation caused by $\mathrm{CO}_{2}$ (Supplementary Figure S1). Finally, the layered ground acts as a high-pass filter due to compliance effects: the higher the frequency the higher the air-to-ground conversion coefficient (Section 4.2.2, Figures 1 and A3). The cutoff associated with this air-to-ground conversion effect can explain why the frequencies of the selected monotone SEIS events never fall below $0.3 \mathrm{~Hz}$ (Figure 7). 
Additionally, it is known that infrasound trapped in narrow surface waveguides are expected to have a dispersive pattern (Negraru \& Herrin, 2009). However, the pressure perturbations linked to the SEIS events are extremely low and could not have been recorded by APSS (Section 5.2). This has two consequences. First, contrary to Earth (Herrin et al., 2006; Negraru \& Herrin, 2009), we cannot observe directly the dispersive pattern in pressure records. Second, the subsequent ground motion is particularly low and high-pass filtered by compliance effects (Section 4.2.2), causing the dispersive pattern to disappear in SEIS' noise level (at low and high frequencies, see Supplementary Figure S7). This explains why these events appear to be monotone in SEIS records. Events with high enough amplitudes to be recorded on APSS will be necessary to confirm this hypothesis.

Using ray tracing simulations in several atmospheric conditions, we can suggest directions favorable to the infrasonic excitation of the nocturnal waveguide. In the early days of the operational phase of the InSight mission (e.g., for the S0105a event) it is straight Southward, then progressively turns to Eastward (for S0133a, and S0152a) and finally straight Eastward (for S0185a, S0189a, S0234c, and S0251a).

In conclusion, we have demonstrated that the S0133a and S0189a seismic events are caused by infrasonic waves trapped in the nocturnal surface waveguide. The other considered events (Table 1) do not have a significant enough signal-to-noise ratio to yield satisfying results. Nonetheless, the rationale developed in this paper can be applied to them and future events, to demonstrate which are caused by infrasound and which are not.

The actual source of atmospheric infrasound still remains to be identified. For some events, gravity wave activity suggests that atmospheric phenomena might be the origin of the generation of infrasound (Section 5.3). Unfortunately, this is not clear for all events. Because of this, further work needs to be put in determining the incoming direction of the guided infrasound, hopefully pinpointing a direction for the search of source events.

The back-azimuth of incoming infrasound can be inferred by studying the polarization of ground movements (Section 4.2, Figure 4) and/or wind recorded by APSS' TWINS instrument. If infrasonic waves are also recorded by APSS, one can use existing algorithms for seismo-acoustic sensor pairs (McKee et al., 2018). If infrasound is associated with a gravity wave train, and wind oscillations are also detected, a source distance may even be estimated (Section 5.3).

\section{Appendix A Validation of the Air-to-Ground Conversion Modes us- ing SPECFEM-DG}

In this Appendix, we validate the capability of SPECFEM-DG in reproducing the various air-to-ground conversion modes: wave-wave transmission (based on Snell's law), evanescent ground motion (Woods et al., 2015), and compliance effects (Sorrells et al., 1971; Kenda et al., 2017, 2020). We use two idealized simulations.

In the first one, the sub-surface is chosen as a single layer hard soil, with $\rho=$ $3100 \mathrm{~kg} / \mathrm{m}^{3}, v_{\mathrm{p}}=5400 \mathrm{~m} / \mathrm{s}$, and $v_{\mathrm{s}}=3117 \mathrm{~m} / \mathrm{s}$. The atmospheric model is chosen as a Mars Climate Database (MCD) output (Forget et al., 1999; Millour et al., 2018) computed for InSight sol 0, $20 \mathrm{~h}$ LMST, and is presented in Supplementary Figure S1. The source is chosen as an atmospheric excitation (Eq. (12)) at $z_{0}=12 \mathrm{~km}$ altitude, with fundamental frequency $f_{0}=3 \mathrm{~Hz}$. Recording stations are distributed along the fluid/solid interface, recording both air pressure perturbation $\Delta P$ and ground velocities $V_{x, z}$ to be able to compute conversion ratios. Figure A1 is a snapshot of the simulation, illustrating the setup. 


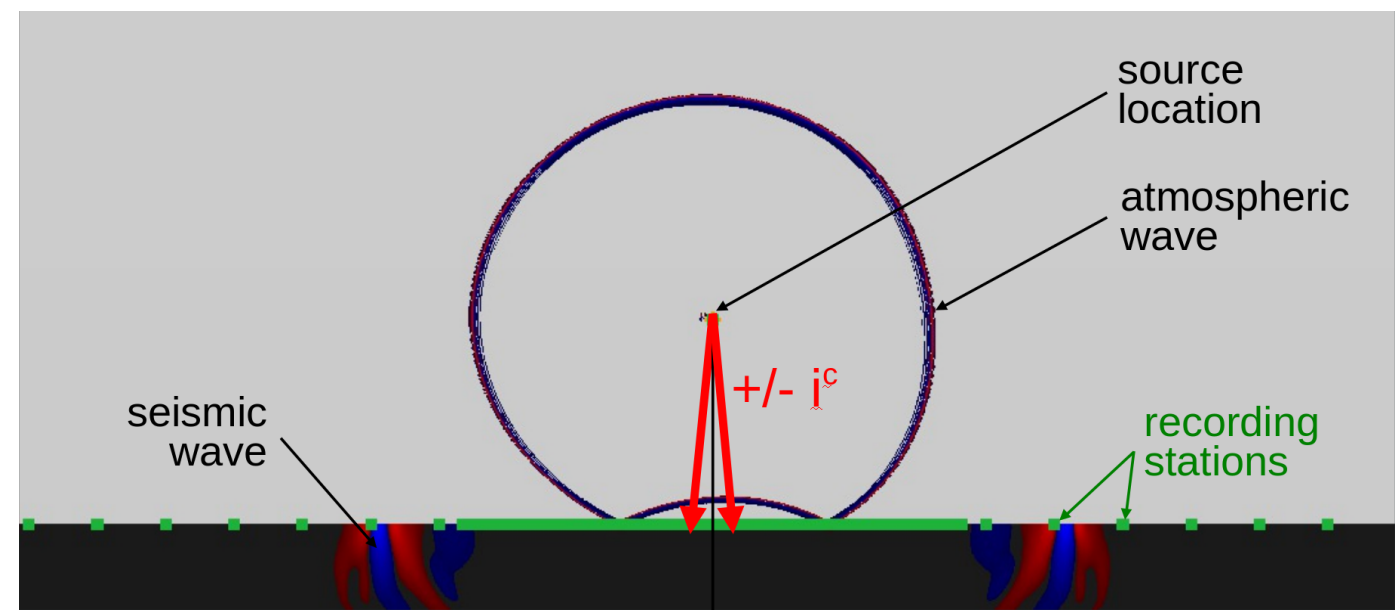

Figure A1. Snapshot of the simulation for validating conversion modes. Dark gray is the ground, light gray is the atmosphere. Shades of red and blue encode values (respectively positive and negative) and represent vertical ground motion in the solid domain, and pressure perturbation in the fluid domain; the scale is saturated at $\pm 1 \%$ maximum amplitude. Green dots represent the recording stations. Black arrows indicate key features. The two red arrows highlight the seismic critical angle $i^{\mathrm{c}}$ (Eq. (5)).

The seismic critical angle - over which acoustic waves cannot be transmitted to seismic waves - can be obtained from Snell's law (Eq. (5)). For this setup, $i^{\mathrm{c}}=$ $\arcsin \left(c_{\mathrm{s}} / v_{\mathrm{s}}\right) \simeq 4.3^{\circ}$. This translates to a critical distance (at which the acoustic incidence angle $\left.i=i^{\mathrm{c}}\right)$ of:

$$
d^{\mathrm{c}}=z_{0} \tan \left(\arcsin \left(c / v_{\mathrm{s}}\right)\right) \simeq 902 \mathrm{~m} .
$$

The wave-wave transmission conversion coefficient at vertical incidence is, from impedance relations (Aki \& Richards, 2002):

$$
V_{z} / \Delta P \simeq\left(\rho^{\mathrm{s}} v_{\mathrm{p}}\right)^{-1} \simeq 5.97 \times 10^{-8}(\mathrm{~m} / \mathrm{s}) / \mathrm{Pa},
$$

where $V_{z}$ is the vertical velocity, and $\Delta P$ is the pressure perturbation. The compliance conversion coefficients into this mono-layer soil are simply (Section 2.3.2):

$$
\begin{aligned}
& V_{z} / \Delta P \simeq 6.12 \times 10^{-9}(\mathrm{~m} / \mathrm{s}) / \mathrm{Pa} \\
& V_{h} / \Delta P \simeq 2.04 \times 10^{-9}(\mathrm{~m} / \mathrm{s}) / \mathrm{Pa},
\end{aligned}
$$

where $V_{h}$ is the horizontal velocity.

The air-to-ground conversion ratios were computed and compared to those theoretical values. Figure A2 presents the results. We perfectly retrieve the wave-wave coefficient when under the critical distance $\left(\left|x-x_{0}\right|<d^{c}\right)$, and tend toward the compliance coefficient when $\left|x-x_{0}\right| \gg d^{\mathrm{c}}$. The transition between the two $-i . e$. , incident angles $i$ such that $i^{\mathrm{c}}<i<90^{\circ}$ - is smooth due to the transmission of energy to an evanescent ground motion mode (Woods et al., 2015, e.g., their Figure 9).

Furthermore, we retrieve the fact the $V_{z}, V_{h}$, and $\Delta P$ are all in phase during the wave-wave conversion regime. In the compliance regime, we retrieve the relations described in Section 2.3.2: $V_{z}$ is $90^{\circ}$ phase shifted with respect to $V_{h}$ and $\Delta P$, and $V_{h}$ and $\Delta P$ are in phase.

For more realistic layered models, the conversion coefficients are more complicated to compute and depend on frequency, which makes an exhaustive comparison 


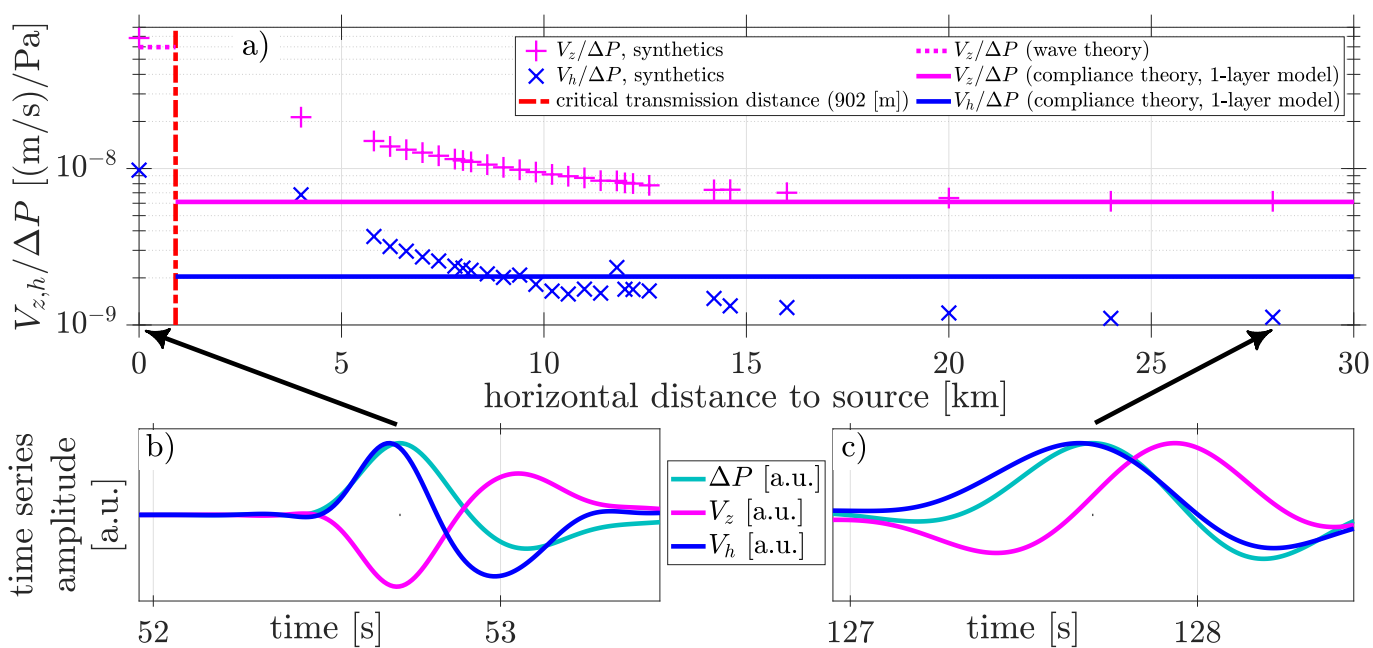

Figure A2. Validation of SPECFEM-DG for air-to-ground conversion. (a) $V_{z} / \Delta P$ (magenta) and $V_{h} / \Delta P$ (blue) ratios, as function of horizontal distance to the source $\left|x-x_{0}\right|$, based on SPECFEM-DG synthetics. The red dashed line represents the critical distance $d^{c}$ for the chosen simulation setup (Eq. (A1)). The magenta dotted line (top left, only plotted to the left of the red dashed line i.e., for distances $<d^{\mathrm{c}}$ ) is the theoretical wave-wave transmission conversion coefficient, computed from impedance theory. The two solid lines (magenta and blue, only plotted to the right of the red dashed line i.e., for distances $>\quad d^{c}$ ) represent the theoretical compliance conversion coefficients (in the $90^{\circ}$ incidence limit). They are computed from compliance theory for a mono-layer soil (Eq. A3). (b) and (c): selected $\left(P, V_{z}, V_{h}\right)$ synthetic time series in arbitrary units, showing the respective phase shifts, (b) at $\left|x-x_{0}\right|=0$, (c) at $\left|x-x_{0}\right|=28 \mathrm{~km}$.

study somewhat more complicated. However, performing a validation on an example case is possible. Consequently, the second full-wave simulation for validation has been performed. The setup is exactly the one presented before, only with a different ground model. Figure A3 presents the chosen ground model, the theoretical multilayer frequency-dependent compliance coefficients (Kenda et al., 2017, 2020), and the compliance ratios computed from this full-wave simulation.

The synthetic signals at the presented horizontal distances remain mostly and consistently monotone at $\simeq 0.4 \mathrm{~Hz}$, justifying the choice of that frequency for the theoretical compliance values. That being said, the agreement is again excellent, highlighting the fact that SPECFEM-DG is reproducing the air-to-ground compliance effects truthfully even on layered soil.

\section{Appendix B Derivation of the Compliance Relations Linking Pressure Perturbations to Ground Motion}

In this Appendix, we derive the relations linking the pressure field to the elastic response of the surface. This is relying heavily on the works of Sorrells (1971), Kenda et al. (2017), and Kenda et al. (2020).

Following the developments of the main paper, the $x$-axis is chosen positive in the direction of propagation of the pressure fluctuation, and the $z$-axis is chosen positive upwards. 

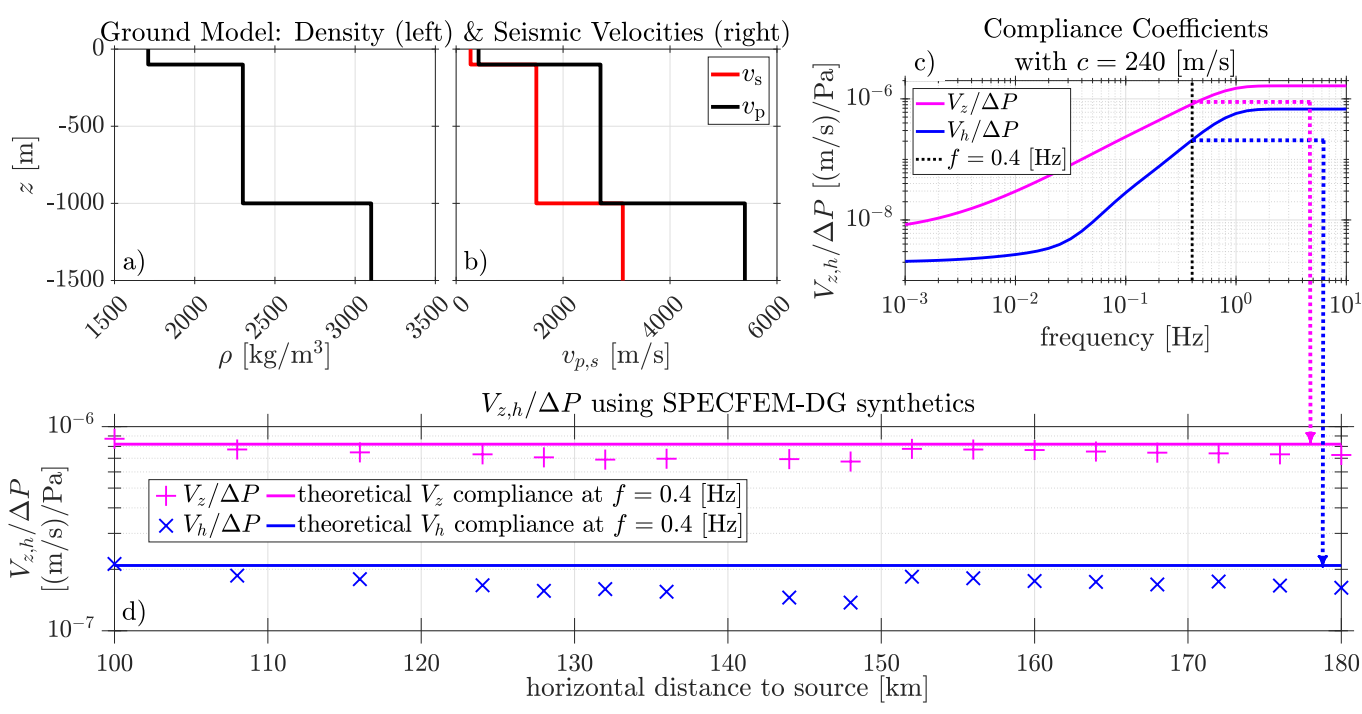

Figure A3. Validation of SPECFEM-DG for the air-to-ground compliance conversion mode. (a) and (b) Ground model density and seismic velocities. (c) Theoretical compliance coefficients for this layered ground (Kenda et al., 2017, 2020), with the last layer taken as an infinite halfspace. The vertical dotted line highlights $f=0.4 \mathrm{~Hz}$. d) $V_{z} / \Delta P$ (magenta) and $V_{h} / \Delta P$ (blue) ratios, as function of horizontal distance to the source $\left|x-x_{0}\right|$, based on SPECFEM-DG synthetics. The two solid lines (magenta and blue) represent the theoretical compliance conversion coefficients at $0.4 \mathrm{~Hz}$, taken from panel c. By choosing large horizontal distances to the source, we ensure we are at incidences $i \rightarrow 90^{\circ}$ and thus not in the evanescent regime anymore (Woods et al., 2015).

We start by splitting a static pressure field depending only on one horizontal component only (say, $x), \Delta P(x)$, into its spatial spectrum:

$$
\Delta P(x)=\int_{-\infty}^{+\infty} \Delta P\left(k_{x}\right) e^{i k x} \mathrm{~d} k_{x},
$$

with $k_{x}$ the wavenumber in the $x$ direction, and $\Delta P\left(k_{x}\right)$ the spectrum component along the wavenumber $k_{x}$. Following (Sorrells, 1971; Kenda et al., 2017, 2020), we treat each spectral component separately.

For each spectral component, the elastic response of the ground at the boundary (at the surface) to $\Delta P\left(k_{x}\right)$ is tied to the elastic properties. Quantitatively, spectral components of the vertical surface displacement $U_{z}$ and horizontal surface displacement $U_{x}$ are given by (Sorrells, 1971; Kenda et al., 2017, 2020):

$$
\begin{gathered}
U_{z}\left(k_{x}\right)=-\frac{1}{k_{x}}\left(\frac{\lambda+2 \mu}{2 \mu(\lambda+\mu)}\right) \Delta P\left(k_{x}\right)=-\frac{1}{k_{x}}\left(\frac{2\left(1-\nu^{2}\right)}{\mathrm{E}}\right) \Delta P\left(k_{x}\right), \\
U_{x}\left(k_{x}\right)=\frac{i}{k_{x}}\left(\frac{\mu}{2 \mu(\lambda+\mu)}\right) \Delta P\left(k_{x}\right)=\frac{i}{k_{x}}\left(\frac{(1+\nu)(1-2 \nu)}{\mathrm{E}}\right) \Delta P\left(k_{x}\right),
\end{gathered}
$$

where $\lambda$ is the first Lamé parameter, $\mu$ is the second Lamé parameter, $\nu$ is the Poisson ratio, and $\mathrm{E}$ is the Young modulus of the elastic half-space.

Now considering a propagating pressure perturbation, $\Delta P(x, t)$, and following Sorrells (1971) by assuming the perturbation is propagating at some horizontal speed $c$, one has that:

$$
\Delta P(x, t)=\Delta P(x-c t),
$$


and the wavenumber of the fluctuation satisfies:

$$
k_{x}=\frac{\omega}{c},
$$

where $\omega=2 \pi f$ is the pulsation (angular frequency). Note that the spectral dependence in $k_{x}$ can now rather be expressed as a more convenient dependence in $f$.

Combining Eq. (B2) with Eq. (B5) yields:

$$
U_{z}(f)=-\frac{c}{2 \pi f}\left(\frac{2\left(1-\nu^{2}\right)}{\mathrm{E}}\right) \Delta P(f),
$$

and combining Eq. (B3) with Eq. (B5) yields:

$$
U_{x}(f)=\frac{i c}{2 \pi f}\left(\frac{(1+\nu)(1-2 \nu)}{\mathrm{E}}\right) \Delta P(f) .
$$

Finally, derivating in the spectral domain (multiplying by $i \omega=2 i \pi f$ ) yields the components of surface velocity:

$$
\begin{gathered}
V_{z}(f)=-2 i c \frac{1-\nu^{2}}{\mathrm{E}} \Delta P(f), \\
V_{x}(f)=-c \frac{(1+\nu)(1-2 \nu)}{\mathrm{E}} \Delta P(f) .
\end{gathered}
$$

In the developments of the main paper, since these responses are the true surface responses, we renamed them in Section 2.3.2 as inertial: $V_{z}{ }^{\mathrm{i}}(f):=V_{z}(f)$ and $V_{h}{ }^{\mathrm{i}}(f):=$ $V_{x}(f)$.

The computation of the tilt $\theta$ in the direction of propagation (here, $x$ ) stems from the fact that it is defined from the vertical displacement: $\tan \theta=\partial U_{z} / \partial x$ (Sorrells, 1971). Consequently, going back and multiplying Eq. (B2) by $i k_{x}$ yields:

$$
[\tan \theta]\left(k_{x}\right)=-i\left(\frac{\lambda+2 \mu}{2 \mu(\lambda+\mu)}\right) \Delta P\left(k_{x}\right)=-i\left(\frac{2\left(1-\nu^{2}\right)}{\mathrm{E}}\right) \Delta P\left(k_{x}\right) .
$$

We are however interested in the apparent horizontal motion caused by tilt, rather than the tilt itself. We recall (see Section 2.3.2) that for SEIS lying on the ground, a tilt of the ground will project local gravity $g$ onto the horizontal components (Sorrells, 1971; Kenda et al., 2020). Consequently, the tilt $\theta$ and the apparent tilt-induced acceleration $A_{x}^{\mathrm{t}}$ are related by (making use of Eq. (B10)):

$$
A_{x}^{\mathrm{t}}\left(k_{x}\right)=-g \sin \left(\theta\left(k_{x}\right)\right)=-g \sin \left(\arctan \left(-2 i \frac{\left(1-\nu^{2}\right)}{\mathrm{E}} \Delta P\left(k_{x}\right)\right)\right),
$$

where $g$ is the local gravity. Since tilts are assumed to be small with the usual ground models (Sorrells, 1971), and since $\sin (\arctan (x)) \simeq x$ for $x \rightarrow 0$, we simplify Eq. (B11) and obtain (Kenda et al., 2017, 2020):

$$
A_{x}^{\mathrm{t}}\left(k_{x}\right)=2 i g \frac{\left(1-\nu^{2}\right)}{\mathrm{E}} \Delta P\left(k_{x}\right) .
$$

Integrating Eq. (B12) in the spectral domain (dividing by $i \omega$ ), one ends up with the apparent, tilt-induced, horizontal ground velocity:

$$
V_{x}^{\mathrm{t}}\left(k_{x}\right)=\frac{2 g}{\omega} \frac{\left(1-\nu^{2}\right)}{\mathrm{E}} \Delta P\left(k_{x}\right) .
$$

Recalling we are in the case of a pressure perturbation propagating at some horizontal speed, Eq. (B5) holds, and we can finally rewrite Eq. (B13) in terms of frequency components:

$$
V_{x}^{\mathrm{t}}(f)=\frac{g}{\pi f} \frac{\left(1-\nu^{2}\right)}{\mathrm{E}} \Delta P(f),
$$


which is renamed $V_{h}^{\mathrm{t}}$ in Section 2.3.2.

To summarize, the inertial vertical and horizontal surface velocities are linked to pressure perturbations by Eqs. (B8)-(B9) (i.e., Eqs. (6)-(7)), while instrument tilt effects are linked to pressure perturbations by Eq. (B14) (i.e., Eq. (8)).

\section{Acronyms}

APSS Auxiliary Payload Sensor Suite (InSight's)

CALMIP CALcul en MIdi-Pyrénées (HPC cluster)

CNES Centre National d'études Spatiales

CNRS Centre National de la Recherche Scientifique

DLR Deutsches Zentrum für Luft- und Raumfahrt e.V.

ETHZ Eidgenssische Technische Hochschule Zürich

HPC High Performance Computing

ISAE-Supaero Institut Supérieur de l'Aéronautique et de l'Espace - Supaero

InSight Interior exploration using Seismic Investigations, Geodesy and Heat Transport

IPGP Institut de Physique du Globe de Paris

IRIS-DMC Incorporated Research Institutions for Seismology - Data Management Center

JPL Jet Propulsion Laboratory (NASA's)

LMST Local Mars Solar Time

LPG Laboratoire de Planétologie et Géodynamique

MSDS Mars SEIS Data Service

MSFC Marshall Space Flight Center

MPS-MPG Max Planck institute for Solar System research (Max Plank Gesellschaft)

MQS Mars Quake Service

NASA National Aeronautics and Space Administration

PDS Planetary Data System (NASA's)

SEIS Seismic Experiment for Interior Structures (InSight's)

SISMOC SeIS on Mars Operation Center (CNES')

SSO Swiss Space Office

TGCC Très Grand Centre de Calcul (HPC cluster)

TWINS Temperature and WINd Sensor

UKSA United Kingdom Space Agency

\section{Acknowledgments}

This study is InSight contribution number 127. The authors thank the TGCC (Paris, France, project GENCI gen10476) and CALMIP (Toulouse, France, project \#p1404) computing centers for HPC resources. The authors acknowledge both the "Direction Générale de l'Armement" (French Department of Defence) and the "Région Occitanie" for funding the $\mathrm{PhD}$ grant of Léo Martire, as well as the CNES for the team's financial support. The authors acknowledge two anonymous reviewers for their constructive remarks. The authors also gratefully thank Balthasar Kenda for fruitful discussions and crucial help with the modeling of ground compliance. We also wish to thank Nicolas Compaire, Guénolé Mainsant, and Baptiste Pinot for performing the deticking and the deglitching of SEIS' data. We acknowledge NASA, CNES, partner agencies and institutions (UKSA, SSO, DLR, JPL, IPGP-CNRS, ETHZ, ICL, MPS-MPG, LPG, MSFC) and the operators of JPL, SISMOC, MSDS, IRIS-DMC and PDS for providing SEED SEIS data. SEIS data is curated by InSight's Mars SEIS Data Ser- 
vice (InSight Mars SEIS Data Service, 2019) and is available at https://doi.org/ 10.18715/SEIS. INSIGHT .XB \_2016. The direct link to InSight's APSS data archive at the Planetary Data System Atmospheres node is https://atmos.nmsu.edu/data_and _services/atmospheres_data/INSIGHT/insight.html (Banfield et al., 2020). The catalog of events is curated by InSight's Marsquake Service (InSight Marsquake Service, 2020) and is available at https://doi.org/10.12686/a6. For this study, we performed simulations using SPECFEM-DG (Brissaud, 2017) as well as WASP-3D (Dessa et al., 2005). The configurations of these simulations are detailed in Section 4. The Martian sunset times in Table 1 are computed using the pyMarsTime package (https://github.com/eelsirhc/pyMarsTime, implementing the algorithm by Allison (1997) and Allison and McEwen (2000)). The derived data related to this paper are stored online (Martire et al., 2020), and available at https://doi.org/ 10.5281/zenodo. 3744832 .

\section{References}

Aki, K., \& Richards, P. G. (2002). Quantitative Seismology, 2nd Edition (Second ed.). Sausalito, California 94965, USA: University Science Books.

Allison, M. (1997, 8). Accurate analytic representations of solar time and seasons on Mars with applications to the Pathfinder/Surveyor missions. Geophysical Research Letters, 24(16), 1967-1970. doi: 10.1029/97GL01950

Allison, M., \& McEwen, M. (2000, 2). A post-Pathfinder evaluation of areocentric solar coordinates with improved timing recipes for Mars seasonal/diurnal climate studies. Planetary and Space Science, 48(2-3), 215-235. doi: 10.1016/S0032-0633(99)00092-6

Assink, J. D. (2012). Infrasound as upper atmospheric monitor (Unpublished doctoral dissertation). University of Mississippi.

Banfield, D. J., Rodriguez-Manfredi, J. A., Russell, C. T., Rowe, K. M., Leneman, D., Lai, H. R., .. Banerdt, W. B. $\quad(2019,2) . \quad$ InSight Auxiliary Payload Sensor Suite (APSS). $\quad$ Space Science Reviews, 215(1), $4 . \quad$ doi: 10.1007/s11214-018-0570-x

Banfield, D. J., Spiga, A., Newman, C., Forget, F., Lemmon, M. T., Lorenz, R. D., ... Banerdt, W. B. (2020, 2). The atmosphere of Mars as observed by InSight. Nature Geoscience. doi: 10.1038/s41561-020-0534-0

Bass, H. E., \& Chambers, J. P. (2001). Absorption of sound in the Martian atmosphere. The Journal of the Acoustical Society of America, 109(6), 3069-3071. doi: $10.1121 / 1.1365424$

Bedard, A. J. (2005). Low-frequency atmospheric acoustic energy associated with vortices produced by thunderstorms. Monthly Weather Review, 133(1), 241263. doi: 10.1175/MWR-2851.1

Brissaud, Q. (2017). Modélisation numérique des ondes atmosphériques issues des couplages solide/océan/atmosphère et applications (Unpublished doctoral dissertation). Université Toulouse 3 Paul Sabatier.

Brissaud, Q., Martin, R., Garcia, R. F., \& Komatitsch, D. (2017). Hybrid Galerkin numerical modelling of elastodynamics and compressible Navier-Stokes couplings: Applications to seismo-gravito acoustic waves. Geophysical Journal International, 210(2), 1047-1069. doi: 10.1093/gji/ggx185

Campus, P., \& Christie, D. R. (2009). Worldwide observations of infrasonic waves. In A. Le Pichon, E. Blanc, \& A. Hauchecorne (Eds.), Infrasound monitoring for atmospheric studies (pp. 185-234). Dordrecht: Springer Netherlands. doi: 10.1007/978-1-4020-9508-5_6

Campus, P., \& Christie, D. R. (2010). Worldwide observations of infrasonic waves. In Infrasound monitoring for atmospheric studies (pp. 185-234). Springer.

Carpenter, M. H., \& Kennedy, C. A. (1994). Fourth-Order 2N-Storage Runge-Kutta Schemes. Nasa Technical Memorandum, 109112, 1-26. 
Daubar, I., Lognonné, P., Teanby, N. A., Miljkovic, K., Stevanović, J., Vaubaillon, J., ... others (2018). Impact-seismic investigations of the insight mission. Space Science Reviews, $214(8), 132$.

de Groot-Hedlin, C. D. $\quad(2017,10)$. Infrasound propagation in tropospheric ducts and acoustic shadow zones. The Journal of the Acoustical Society of America, 142(4), 1816-1827. doi: 10.1121/1.5005889

Delage, P., Karakostas, F., Dhemaied, A., Belmokhtar, M., Lognonné, P. H., Golombek, M. P., .. Banerdt, W. B. (2017). An Investigation of the Mechanical Properties of Some Martian Regolith Simulants with Respect to the Surface Properties at the InSight Mission Landing Site. Space Science Reviews, 211(1-4), 191-213. doi: 10.1007/s11214-017-0339-7

Dessa, J. X., Virieux, J., \& Lambotte, S. (2005). Infrasound modeling in a spherical heterogeneous atmosphere. Geophysical Research Letters, 32(12), 1-5. doi: 10 .1029/2005GL022867

Drob, D. P., Picone, J. M., \& Garcés, M. (2003). Global morphology of infrasound propagation. Journal of Geophysical Research (Atmospheres), 108(D21), 4680. doi: 10.1029/2002JD003307

Evers, L. G., \& Haak, H. W. (2009). The characteristics of infrasound, its propagation and some early history. In A. Le Pichon, E. Blanc, \& A. Hauchecorne (Eds.), Infrasound monitoring for atmospheric studies (pp. 3-27). Dordrecht: Springer Netherlands. doi: 10.1007/978-1-4020-9508-5_1

Forget, F., Hourdin, F., Fournier, R., Hourdin, C., Talagrand, O., Collins, M., ... Huot, J.-P. $\quad(1999,10)$. Improved general circulation models of the Martian atmosphere from the surface to above $80 \mathrm{~km}$. Journal of Geophysical Research E: Planets, 104(E10), 24155-24175. doi: 10.1029/1999JE001025

Garcés, M. M., Willis, M., Hetzer, C., Le Pichon, A., \& Drob, D. P. (2004, 10). On using ocean swells for continuous infrasonic measurements of winds and temperature in the lower, middle, and upper atmosphere. Geophysical Research Letters, 31 (19), L19304. doi: 10.1029/2004GL020696

Garcia, R. F., Brissaud, Q., Rolland, L. M., Martin, R., Komatitsch, D., Spiga, A., ... Banerdt, W. B. $\quad(2017,10)$. Finite-Difference Modeling of Acoustic and Gravity Wave Propagation in Mars Atmosphere: Application to Infrasounds Emitted by Meteor Impacts. Space Science Reviews, 211(1-4), 547-570. doi: 10.1007/s11214-016-0324-6

Garcia, R. F., Kenda, B., Kawamura, T., Spiga, A., Murdoch, N., Lognonné, P. H., ... Banerdt, W. B. (2020). Pressure Effects on the SEIS-InSight Instrument, Improvement of Seismic Records, and Characterization of Long Period Atmospheric Waves From Ground Displacements. Journal of Geophysical Research: Planets, e2019JE006278. doi: 10.1029/2019JE006278

Garratt, J. R. (1992). The Atmospheric Boundary Layer. Earth-Science Reviews, $37(1-2), 89-134$

Giardini, D., Lognonné, P. H., Banerdt, W. B., Pike, W. T., Christensen, U., Ceylan, S., .. Yana, C. $(2020,2)$. The seismicity of Mars. Nature Geoscience, in press. doi: 10.1038/s41561-020-0539-8

Godin, O. A., Yu. Mikhin, D., \& Molchanov, S. Y. (1993). Effective sound speed approximation in the acoustics of moving media. Izvestiya-Atmospheric \& Oceanic Physics, 29(2), 179-186.

Gossard, E. E., \& Hooke, W. H. (1975). Waves in the atmosphere: Atmospheric infrasound and gravity waves - Their generation and propagation (first ed., Vol. 2). Elsevier Scientific Publishing.

Haberle, R., Houben, H. C., Hertenstein, R., \& Herdtle, T. (1993, june). A boundary layer model for Mars: Comparison with Viking Lander and entry data. Journal of the Atmospheric Sciences, 50, 1544-1559.

Heavens, N. G., Kass, D. M., Shirley, J. H., Piqueux, S., \& Cantor, B. A. (2019) An Observational Overview of Dusty Deep Convection in Martian Dust 
Storms. Journal of Atmospheric Sciences, 76(11), 3299-3326. doi: 10.1175/JAS-D-19-0042.1

Herrin, E. T., Kim, T. S., \& Stump, B. W. $\quad(2006,4) . \quad$ Evidence for an infrasound waveguide. Geophysical Research Letters, 33(7), L07815. doi: 10.1029/2005GL025491

Howe, M. S. (2002). Lectures on the Theory of Vortex-Sound. In Sound-flow interactions (pp. 31-111).

InSight Mars SEIS Data Service. (2019). SEIS raw data, Insight Mission. IPGP, JPL, CNES, ETHZ, ICL, MPS, ISAE-Supaero, LPG, MFSC. doi: 10.18715/ SEIS.INSIGHT.XB $\{\backslash-\} 2016$

InSight Marsquake Service. (2020). Mars Seismic Catalogue, InSight Mission; V1 2/1/2020. ETHZ, IPGP, JPL, ICL, ISAE-Supaero, MPS, Univ. Bristol. doi: $10.12686 / \mathrm{A} 6$

Kenda, B., Garcia, R. F., Drilleau, M., Kawamura, T., Murdoch, N., Compaire, N., .. Spohn, T. (2020). Subsurface structure at the InSight landing site from compliance measurements by SEIS and APSS. Journal of Geophysical Research: Planets.

Kenda, B., Lognonné, P. H., Spiga, A., Kawamura, T., Kedar, S., Banerdt, W. B., ... Golombek, M. P. (2017). Modeling of Ground Deformation and Shallow Surface Waves Generated by Martian Dust Devils and Perspectives for NearSurface Structure Inversion. $\quad$ Space Science Reviews, 211(1-4), 501-524. doi: 10.1007/s11214-017-0378-0

Komatitsch, D., \& Tromp, J. (1999). Introduction to the spectral element method for three-dimensional seismic wave propagation. Geophysical Journal International, 139, 806-822.

Komatitsch, D., \& Vilotte, J.-P. (1998). The Spectral Element Method : An Efficient Tool to Simulate the Seismic Response of 2D and 3D Geological Structures. Bulletin of the Seismological Society of America, 88(2), 368-392. doi: 10.1.1.716.9673

Krishnamoorthy, S., Komjathy, A., Pauken, M. T., Cutts, J. A., Garcia, R. F., Mimoun, D., ... Bowman, D. C. (2018). Detection of Artificially Generated Seismic Signals using Balloon-borne Infrasound Sensors. Geophysical Research Letters. doi: 10.1002/2018GL077481

Krishnamoorthy, S., Lai, V. H., Komjathy, A., Pauken, M. T., Cutts, J. A., Garcia, R. F., .. Cadu, A. (2019). Aerial Seismology Using Balloon-Based Barometers. IEEE Transactions on Geoscience and Remote Sensing, 1-11. doi: 10.1109/TGRS.2019.2931831

Larson, R. J., Craine, L. B., Thomas, J. E., \& Wilson, C. R. $\quad(1971,9)$. Correlation of Winds and Geographic Features with Production of Certain Infrasonic Signals in the Atmosphere. Geophysical Journal of the Royal Astronomical Society, 26(1-4), 201-214. doi: 10.1111/j.1365-246X.1971.tb03395.x

Le Pichon, A., Blanc, E., \& Drob, D. P. (2005). Probing high-altitude winds using infrasound. Journal of Geophysical Research: Atmospheres, 110(20), 1-4. doi: 10.1029/2005JD006020

Lognonné, P. H., Banerdt, W. B., Giardini, D., Pike, W. T., Christensen, U., Laudet, P., ... Wookey, J. $\quad(2019,2)$. SEIS: Insights Seismic Experiment for Internal Structure of Mars. $\quad$ Space Science Reviews, 215(1), $12 . \quad$ doi: 10.1007/s11214-018-0574-6

Lognonné, P. H., Banerdt, W. B., Pike, W. T., Giardini, D., Christensen, U., Garcia, R. F., .. U., C. $\quad(2020,2)$ Constraints on the shallow elastic and anelastic structure of Mars from InSight seismic data. Nature Geoscience, in press(February). doi: 10.1038/s41561-020-0536-y

Lorenz, R. D., \& Christie, D. R. $\quad(2015,3)$. Dust devil signatures in infrasound records of the International Monitoring System. Geophysical Research Letters, 42(6), 2009-2014. doi: 10.1002/2015GL063237 
Martire, L., Brissaud, Q., Lai, V. H., Garcia, R. F., Martin, R., Krishnamoorthy, S., ... Sournac, A. $(2018,11)$. Numerical Simulation of the Atmospheric Signature of Artificial and Natural Seismic Events.

Geophysical Research Letters, 45(21), 085-12. doi: 10.1029/2018GL080485

Martire, L., Garcia, R. F., Rolland, L., Spiga, A., Lognonné, P., Banfield, D., .. . Martin, R. (2020, 4). Data for "Martian Infrasound: Numerical Modeling and Analysis of InSight's Data". doi: 10.5281/zenodo.3744832

McKee, K., Fee, D., Haney, M. M., Matoza, R. S., \& Lyons, J. (2018). Infrasound Signal Detection and Back Azimuth Estimation Using Ground-Coupled Airwaves on a Seismo-Acoustic Sensor Pair. Journal of Geophysical Research: Solid Earth, 123(8), 6826-6844. doi: 10.1029/2017JB015132

Millour, E., Forget, F., González-Galindo, F., Spiga, A., Lebonnois, S., Lewis, S. R., ... Huot, J.-P. (2018). The Mars Climate Database (version 5.3). In Sae technical paper series (p. 2). doi: 10.4271/2009-01-2395

Morgan, P., Grott, M., Knapmeyer-Endrun, B., Golombek, M. P., Delage, P., Lognonné, P. H., ... Kedar, S. (2018). A Are-Landing Assessment of Regolith Properties at the InSight Landing Site. Space Science Reviews, 214(6). doi: $10.1007 / \mathrm{s} 11214-018-0537-y$

Murdoch, N., Kenda, B., Kawamura, T., Spiga, A., Lognonné, P. H., Mimoun, D., \& Banerdt, W. B. (2017). Estimations of the Seismic Pressure Noise on Mars Determined from Large Eddy Simulations and Demonstration of Pressure Decorrelation Techniques for the Insight Mission.

Space Science Reviews, 211(1-4), 457-483. doi: 10.1007/s11214-017-0343-y

Negraru, P. T., \& Herrin, E. T. (2009, 7). On Infrasound Waveguides and Dispersion. Seismological Research Letters, 80(4), 565-571. doi: 10.1785/gssrl.80.4 .565

Petculescu, A. G., \& Lueptow, R. M. (2007, 2). Atmospheric acoustics of Titan, Mars, Venus, and Earth. Icarus, 186(2), 413-419. doi: 10.1016/j.icarus.2006.09 .014

Savijärvi, H., \& Siili, T. (1993). The Martian slope wind and the nocturnal PBL jet. Journal of the Atmospheric Sciences, 50, 77-88.

Sorrells, G. G. (1971, 9). A Preliminary Investigation into the Relationship between LongPeriod Seismic Noise and Local Fluctuations in the Atmospheric Pressure Field. Geophysical Journal of the Royal Astronomical Society, 26(1-4), 71-82. doi: 10.1111/j.1365-246X.1971.tb03383.x

Sorrells, G. G., McDonald, J. A., Der, Z. A., \& Herrin, E. T. (1971). $\quad$ Earth Motion Caused by Local Atmospheric Pressure Changes. Geophysical Journal of the Royal Astronomical Society, 26(1-4), 83-98. doi: 10.1111/j.1365-246X.1971 .tb03384.x

Spiga, A., Banfield, D. J., Teanby, N. A., Forget, F., Lucas, A., Kenda, B., ... Banerdt, W. B. $\quad(2018,10)$. Atmospheric Science with InSight. Space Science Reviews, 214(7), 109. doi: 10.1007/s11214-018-0543-0

Spiga, A., Hinson, D., Madeleine, J., Navarro, T., Millour, E., Forget, F., \& Montmessin, F. (2017). Snow precipitation on Mars driven by cloud-induced nighttime convection. Nature Geoscience, 10, 652-657. doi: 10.1038/ngeo3008

Tatom, F. B., Knupp, K. R., \& Vitton, S. J. (1995, 2). Tornado Detection Based on Seismic Signal. Journal of Applied Meteorology, 34(2), 572-582. doi: 10.1175/ 1520-0450(1995)034<0572:TDBOSS $\rangle 2.0 . C O ; 2$

Terrana, S., Vilotte, J.-P., \& Guillot, L. (2018). A spectral hybridizable discontinuous Galerkin method for elastic-acoustic wave propagation. Geophysical Journal International, 213(1), 574-602. doi: 10.1093/GJI/GGX557

Tromp, J., Komatitsch, D., \& Liu, Q. (2008). Spectral-element and adjoint methods in seismology (Vol. 3) (No. 1).

Waxler, R. M., Gilbert, K. E., \& Talmadge, C. L. (2008). A theoretical treatment of the long range propagation of impulsive signals under strongly ducted noc- 
turnal conditions. The Journal of the Acoustical Society of America, 124(5), 2742-2754. doi: 10.1121/1.2980520

Whitaker, R. W., \& Norris, D. E. (2008). Infrasound Propagation. In Handbook of signal processing in acoustics (pp. 1497-1519). Springer Science \& Business Media. doi: 10.1007/978-0-387-30441-0\{\-\}82

Williams, J.-P. $\quad(2001,3)$. Acoustic environment of the Martian surface. Journal of Geophysical Research: Planets, 106(E3), 5033-5041. 이 10.1029/ 1999JE001174

Woods, D. C., Bolton, J. S., \& Rhoads, J. F. $\quad(2015,10)$. On the use of evanescent plane waves for low-frequency energy transmission across material interfaces. The Journal of the Acoustical Society of America, 138(4), 2062-2078. doi: $10.1121 / 1.4929692$

Yamamoto, M. Y., Ishihara, Y., Hiramatsu, Y., Kitamura, K., Ueda, M., Shiba, Y., ... Fujita, K. (2011). Detection of acoustic/infrasonic/seismic waves generated by hypersonic re-entry of the HAYABUSA capsule and fragmented parts of the spacecraft. Publications of the Astronomical Society of Japan, 63(5), 971-978. doi: $10.1093 / \mathrm{pasj} / 63.5 .971$ 